\title{
High-speed adaptive wireless body area networks
}

\author{
Miftadi Sudjai ${ }^{1 *} \mathbb{B}$, Le Chung Tran', Farzad Safaei ${ }^{1}$, Tadeusz Wysocki ${ }^{2,3}$ and Son Lam Phung ${ }^{1}$
}

\begin{abstract}
Adaptive ultra-wideband wireless body area network (WBAN) is proposed in this paper as a feasible mobile health platform, offering a high-speed and robust service. It employs a space-time-frequency-coded (STFC) multiband orthogonal frequency division multiplexing ultra-wideband technology. It is designed to improve the average bit error performance for high data rate applications while maintaining a reasonably high throughput in body-toexternal links of a WBAN. The proposed adaptive WBAN comprises two novel components, namely, the frame transmission model and the bit error rate (BER) estimation-based adaptive algorithm. In addition, the optimization of adaptive parameters is conducted to obtain an extra improvement, hence enhancing the performance further. Simulation results show that the proposed optimized adaptive system achieves up to a 2-dB gain over the adaptive WBAN, thus providing up to a 6-dB gain over the non-adaptive WBAN system. This improvement is equivalent to a reduction of the power consumption by up to $37 \%$ compared to the non-optimized adaptive WBAN and up to $75 \%$ compared to the non-adaptive WBAN system, thus extending the battery life and enhancing the reliability of WBAN services significantly.
\end{abstract}

Keywords: Adaptive system, MB-OFDM UWB, STFC, WBAN

\section{Introduction}

Mobile health (mHealth) fosters the advancement of personal and mobile healthcare services. It promises an effective health monitoring system which is potentially capable of reducing the ever-increasing healthcare cost of the ageing society [1]. The current health monitoring systems utilize the inflexible and cumbersome cableconnected instruments which force the patients to visit the doctor or hospital for a health check, and prevent them to move freely. These facts gave us motivation to find a cost-effective and energy-efficient adaptive wireless health monitoring system, which provide the patient a freedom of movement while their health condition is being monitored continuously and remotely by their doctor, thus reducing the necessity and the frequency to visit their doctor or hospital for a health check.

\footnotetext{
* Correspondence: ms917@uow.edu.au

This paper is presented in part at the IEEE International Conference on Communication (ICC), Sydney, Australia, 9-13 June 2014, and at the 48th DGBMT annual conference, Hanover, Germany, 8-10 Oct. 2014.

${ }^{1}$ School of Electrical, Computer, and Telecommunications Engineering (SECTE), University of Wollongong, Northfield Avenue, Wollongong New South Wales 2522, Australia

Full list of author information is available at the end of the article
}

Reliable, tiny, lightweight medical sensors and a robust energy-efficient communication system are keys for the success of mHealth delivery $[1,2]$. Therefore, mHealth services could benefit from the development of advanced wireless body area networks (WBANs). A WBAN consists of wearable and implantable sensors to continuously monitor physiological conditions and feed real-time data wirelessly to the doctor and/or the patient [3]. An impulse radio ultra-wideband (IR-UWB)based WBAN standard has been released by IEEE [3]. However, while it offers low complexity in implementation, it can only support low to medium data rate (few kbps to maximum $15.6 \mathrm{Mbps}$ ) with the bandwidth of $499 \mathrm{MHz}$ [3].

A WBAN platform presents a number of challenges. First, the monitoring and communication systems have to be reliable and accurate. Second, the devices have to be small, light, and highly power efficient to ensure their suitability and longevity. Third, the systems have to provide high capacity to support many sensors and to cater for future bandwidth-hungry applications and services. The multiband orthogonal frequency division multiplexing ultra-wideband (MB-OFDM UWB) technology 
endorsed by WiMedia Alliance [4] offers a high-speed option for the WBAN physical (PHY) layer. It supports very high data rate of up to 1 Gbps with the bandwidth of $528 \mathrm{MHz}$ [4].

To address those issues, in this paper we propose an optimized adaptive STFC MB-OFDM UWB system as a high data rate physical (PHY) layer for a WBAN system. The STFC is employed in order to exploit the diversity of the off-body WBAN channel, which is very diverse in nature. This proposed adaptive algorithm selects a suitable set among three possible sets of modulation, STFC coding rate, and transmit signal power. Each set employs a combination between a BPSK and QPSK, a full-rate Alamouti code or a 3/2-rate Sezginer-Sari code, and a normalized transmit power of $1.5,1.0$, or 0.5 as described in detail in Section 4. The Alamouti code and Sezginer-Sari code are chosen as STFC codes because they offer a full diversity, full rate, and higher rate, for Alamouti code and Sezginer-Sari code, respectively. Moreover, they have a simple decoding process as described later in Section 4.1. The switching between adaptive schemes is determined by two BER thresholds, namely, the lower and upper thresholds. In order to pursue the maximum performance and power reduction, the optimization of adaptive parameters is conducted in this work. Two most important parameters are optimized, i.e., the $f$ factor that defines a portion of the received frame where the BER is measured and the set of adaptive scheme of the rest of the frame is updated, and the $k$ factor which defines a distance between upper and lower BER thresholds of the adaptive algorithm. This optimized adaptive scheme results in a performance improvement by up to $6 \mathrm{~dB}$, meaning a possible $75 \%$ reduction of the total transmitted power, compared to a non-adaptive WBAN.

The major contributions of this paper include:

(i) The proposed BER-based adaptive WBAN system working on a frame-by-frame basis that significantly improves the performance of a WBAN. Including in the proposal are the adaptive WBAN system and transmission model, a novel adaptive algorithm which focuses on adapting the STFC coding rates in combination with modulation and power control, and an analysis of the decoding complexity of the algorithm.

(ii) The optimization of adaptive parameters, namely, the portion of the frame where the BER is measured and the adaptive scheme is updated ( $f$ factor), and the distance between the upper and lower thresholds of the BER metric ( $k$ factor), in order to obtain an extra performance improvement and extra power saving.

(iii) An extension of the original IEEE CM4 UWB WBAN channel model via a linear interpolation, results in a $1^{\circ}$ channel resolution, compared to the original $90^{\circ}$ resolution. This $1^{\circ}$ resolution extended CM4 channel is very important to characterize better the fading channel in the WBAN.

The paper is organized as follows: Section 2 describes related works in this field. Section 3 presents the interpolated CM4 UWB WBAN channel model. Section 4 describes our proposed adaptive WBAN system. The optimization of the $f$ and $k$ parameters is presented in Section 5. Simulation results and discussions are presented in Section 6. Section 7 concludes the paper.

\section{Related works}

Attracted by a wide range of potential applications, such as for entertainment, military, and health care, WBANs have become one of the main research streams during the last several years [1]. The capability of WBAN systems to wirelessly interconnect body-centric devices opens up possibilities of new technology such as implantable and wearable sensors and transceivers to monitor internal organs and health conditions $[1,2]$. To push forward the implementation and commercialization of WBAN systems, IEEE established an IR-UWB-based WBAN standard in 2012 [3]. However, the standard only supports the maximum data rate up to $15.6 \mathrm{Mbps}$ with a bandwidth of $499 \mathrm{MHz}$. It is foreseeable that with the surge of various future bandwidth-hungry applications such as a higher quality video capsule endoscopy, more accurate sensor technology, and larger number on body sensors, will eventually push that capacity to the edge in the near future.

Another competing technology for a WBAN system is MB-OFDM UWB endorsed by WiMedia Alliance [4]. This technology has a more complex hardware implementation compared to IR-UWB; however, it offers much higher data rates up to 1 Gbps. The MB-OFDM UWB system was originally intended for wireless personal area networks (WPAN) and was assessed in fading channels, e.g., by [5]. To enhance the diversity order and improve the performance, a space-time coded multiple input multiple output (MIMO) MB-OFDM UWB system was proposed in [6] and [7]. Further processing in the frequency domain to improve the performance in fading channels was presented in [8]. In a MIMO MB-OFDM UWB system, the performance is greatly determined by the space-time code. A number of possible space-time codes were reported in [9-12]. All above works consider only either a flat-fading or selective-fading channel. None of them consider body-centric channels that have specific characteristics of radio wave propagation in or on the proximity of the human body. Due to the nature of body area channels which are severely dispersive [13] and the need to provide high data rate and robust 
WBAN services, we proposed the use of MB-OFDM UWB as a physical layer for high data rate WBANs, in combination with space-time-frequency-coded MIMO (STFC MIMO) techniques [14]. We demonstrated in [14] that the proposed system, referred to as STFC MBOFDM UWB WBAN, could provide better performance compared to the conventional MB-OFDM UWB with the price of a modest increase in complexity. However, further improvement of the system is necessary to reduce the power consumption.

The WBAN channel models published by IEEE [15] define four different channel models (CM1-CM4), where CM4 is suitable for the body-to-external link and is of interest to this paper. In order to make a more general and flexible channel model, here we modify the IEEE CM4 UWB WBAN channel model by means of linear interpolation. This extended CM4 UWB WBAN channel model will be used to investigate the comparative performance and throughput of the adaptive WBAN system. It is worth to note that recent publications such as [16] provide a more accurate off-body WBAN communication channel model. However, the channel model in this paper is not based on [16] and will be considered in future work.

Adaptive techniques have been employed for numerous applications. One of the early works on adaptive modulation for individual subcarriers of an OFDM system is [17]. It used a BER metric for controlling adaptive selection. However, it considered only BPSK, QPSK, and M-QAM without transmit power control and devoid of exploiting the diversity of OFDM channels. Keller and Hanzo investigated adaptive OFDM with the focus on the trade-off between performance and throughput [18]. They also provided a number of adaptive OFDM techniques and their performance in [19]. Those works mainly focused on gaining a balance between performance and throughput by employing limited number of modulation, i.e., BPSK, QPSK, and 16-QAM without taking advantage of channel diversity through MIMO system. In [20], the authors examined a unified adaptive modulation approach where the data rate, transmitted power, and instantaneous BER were varied to maximize spectral efficiency. While it gives fundamental ideas of combining three aforementioned metrics for channel adaptation, Chung and Goldsmith [20] do not provide a platform to maximize channel diversity and utilize a rate variation by employing MIMO system. Fu, et al. proposed a link adaptation scheme for MB-OFDM UWB systems [21] with a modest performance improvement. A cross-layer adaptive modulation to minimize the transmission energy in wireless sensor networks was proposed in [22]. Yang and Sayrafian-Pour proposed an adaptive modulation, rate, and duty cycle for body area networks (BANs) to mitigate coexisting wireless interference, including from nearby BANs [23]. Its basic principle, however, is reducing transmit power by using a link adaptation scheme in order to maintain a minimum link quality, hence sacrifice the performance. An optimization of switching level based on the received signal strength to select an adaptive scheme for a frame transmission was proposed in [24]. Its optimization works on the minimization of the difference between upper bound performance and the desired performance on frame-to-frame basis. This scheme works well in high SNR. However, in low SNR it works only by disabling the transmission hence sacrifice the capacity to the extent below that of BPSK. Authors in [25] proposed a dual physical layer (PHY) WBAN which focuses on the design of MAC protocol to optimize data forwarding between those PHY to the external networks. However, they only considered energy efficiency and delay parameters as the performance metrics and did not take into account the BER and throughput performance. An adaptive medium access control (MAC) was presented in [26], in which the focus is on an adaptive guard band assignment and sleep/wake up mechanism. This scheme is for the MAC layer and does not consider adaptive scheme for the physical layer. An adaptive power control for a WBAN is proposed in [27] without considering adaptive modulation and STFC coding. Although Sodro et al. [27] provide a significance power saving, they do not exploit the multipath diversity nature of WBAN channel through a MIMO. In other words, adaptive schemes for the WBAN system which are capable to take advantage of a rich diversity of body area channels by utilizing a MIMO technique have been largely unexplored in the literature.

Therefore in [28], we proposed for the first time, a simple but practical, angular body direction-based adaptive algorithm implemented in STFC MB-OFDM UWB WBAN. However, it requires additional hardware and its performance is limited. Hence, our consecutive works [29] proposed a new transmission model and a new adaptive algorithm based on measured BER as metric for adaptation to improve the BER and throughput performance. This paper aims to enhance the works in [29, 30] by more comprehensive approaches, including the extended CM4 UWB WBAN channel model and optimization of its adaptive parameters ( $f$ and $k$ factors).

\section{CM4 channel interpolation}

\subsection{Review of IEEE's CM4 WBAN channel model}

Assume a hub is placed in front of the body (e.g., in torso). This hub acts as a concentrator and collects data from various implantable and wearable body sensors via channel model 2 (CM2) and channel model 3 (CM3) links $[3,15]$. CM2 is a channel model characterizing an implant-to-body surface and implant-to-external links. 
CM3 defines a body surface-to-body surface link for both line of sight (LOS) and non-line of sight (NLOS) links. The hub is connected to external gateway or fixed wireless access point placed in the wall through CM4 channel at the distance $d$. For the sake of simplicity, let us assume the body rotates anti-clockwisely against the wireless access point (WAP). Thus, the CM4 channel characteristics will vary according to the body direction toward the access point. Three adaptive schemes are applied to different channels experienced by the rotating transceiver of the hub on the body toward WAP.

The IEEE's CM4 UWB WBAN channel model proposed for a body surface-to-external WBAN link [13,15] is depicted in Fig. 1. One of its three designated frequency bands is the UWB band $(3.1-10.6 \mathrm{GHz})$. The CM4 channel model takes into account the body directions toward an external transceiver. The CM4 determines different propagation channels for four discrete body directions $0^{\circ}, 90^{\circ}, 180^{\circ}$, and $270^{\circ}$. To make a finer CM4 channel modeling, herein we develop an interpolation of the CM4 with a $1^{\circ}$ resolution, instead of $90^{\circ}$ resolution as above.

The CM4 UWB channel response is characterized by the following power delay profile $[13,15]$ :

$$
h(t)=\sum_{m=0}^{L-1} \alpha_{m} \delta\left(t-\tau_{m}\right)
$$

where $\left|\alpha_{m}\right|^{2}=\Omega_{\mathrm{o}} \mathrm{e}^{-\frac{\tau_{\mathrm{m}}}{\mathrm{F}}-k[1-\delta(m)]} \beta, k=\Delta k\left(\frac{\ln 10}{10}\right), \tau_{o}=d / c$, $d$ is the distance between transmitter (Tx) and receiver $(\mathrm{Rx}), c$ is the velocity of light, $L$ is the number of arrival paths modeled as a Poisson random variable with the mean value of $400 . \beta \sim \log$ normal $(0, \sigma), \alpha_{m}$ is the amplitude of each path, $\tau_{m}$ is timing of path arrivals for $m=$ $1, \ldots, L-1$ which is modeled as a Poisson random process with the arrival rate $\lambda=1 / 0.501251 \mathrm{~ns}, \Gamma$ is an exponential decay factor with $\Omega_{o}$ as the path loss, and $k$ is the $\mathrm{K}$ factor of non-line of sight (NLOS). The CM4 depends on the body direction toward the fixed external transceiver antenna as shown in Table 1 [15].

Realizations of the CM4 UWB WBAN model were provided by the MATLAB code of the appendix of the IEEE P802.15-08-0780-12-0006 document [15]. Their PDP characteristics are depicted in Fig. 2 for the body directions of $0^{\circ}, 90^{\circ}, 180^{\circ}$, and $270^{\circ}$. These PDP were examined as the relative power of the multipath signals arriving at different time delays. These relative powers were calculated from the square absolute of the amplitude of the channel responses of each body direction. Figure $2 \mathrm{a}$ shows that at $0^{\circ}$ direction, the power level at time arrival $\tau_{m}=0 \mathrm{~ns}$ is at the highest, which is dominating the cluster of the multipath signals due to the LOS component of the signal. Figure $2 \mathrm{~b}$ shows an attenuated LOS component $\left(-8.76 \mathrm{~dB}\right.$ at $\left.\tau_{m}=0 \mathrm{~ns}\right)$ at the $90^{\circ}$ direction, which is due to a partial LOS component arrived at the side of the body. At the $180^{\circ}$ direction (the back of the body), the LOS component still exists, though its power level (at $-12.14 \mathrm{~dB})$ is significantly attenuated as shown in Fig. 2c.

Ideally, at the $180^{\circ}$ direction, the LOS component of the signal should vanish completely due to the body shadowing. However, this CM4 UWB WBAN channel realization model showed otherwise, and a residual LOS component still existed. One of possible explanations is that the body did not wholly attenuate the incoming LOS signal, allowing that residual LOS component to appear at the back side of the body (a human body is also a propagation medium or environment). Note that in both $90^{\circ}$ and $180^{\circ}$ directions, clusters of multipath components dominate the PDP. This is because the NLOS signals were dominant at those directions.

It is interesting to note that the worst link is the $270^{\circ}$ body direction (Fig. 2d), rather than $180^{\circ}$ body direction. Despite the fact that there is no explanation from [15],

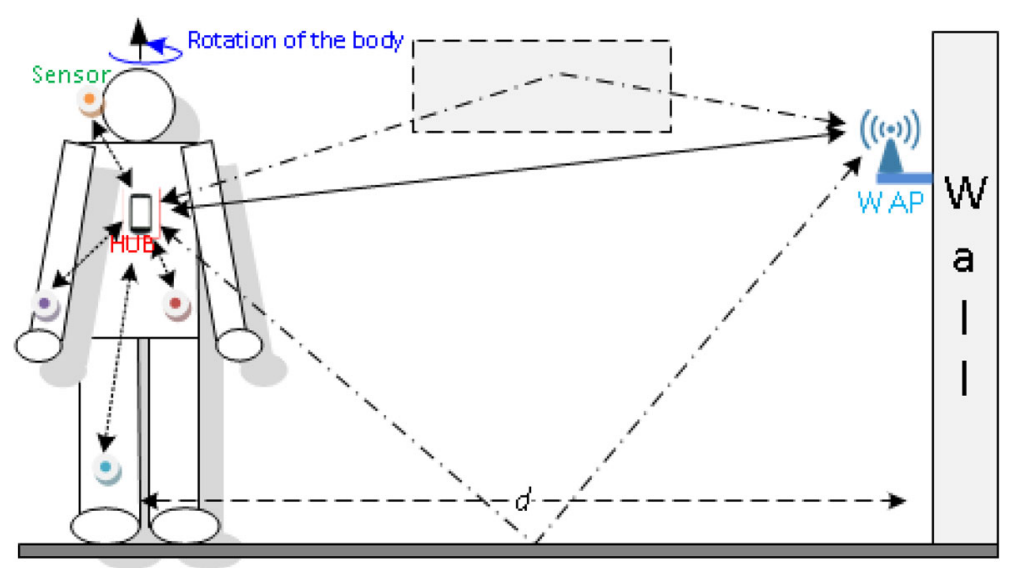

Fig. 1 WBAN channel models and body directions of CM4 
Table 1 Properties of CM4

\begin{tabular}{llll}
\hline Body direction & $\Gamma(\mathrm{ns})$ & $k(\Delta k(\mathrm{~dB}))$ & $\sigma(\mathrm{dB})$ \\
\hline $0^{\circ}$ & 44.6364 & $5.111(22.2)$ & 7.30 \\
$90^{\circ}$ & 54.2868 & $4.348(18.8)$ & 7.08 \\
$180^{\circ}$ & 53.4186 & $3.638(15.8)$ & 7.03 \\
$270^{\circ}$ & 83.9635 & $3.983(17.3)$ & 7.19 \\
\hline
\end{tabular}

this might possibly be due to the changing propagation environments of $270^{\circ}$ and $180^{\circ}$ directions during the measurement campaign.

\subsection{Interpolated CM4 UWB WBAN channel model}

It is noted that the above model only provides a $90^{\circ}$ resolution rather than a $1^{\circ}$ resolution. Furthermore, there is no $1^{\circ}$ resolution channel model available in the literature. The $1^{\circ}$ resolution CM4 channel model is very important to characterize better the fading channel in the WBAN scenario in Fig. 1, thus imitating better the realistic performance of this WBAN system.

Note that the logarithmic value of $\left|\alpha_{m}\right|^{2}$ of Eq. (1), i.e., $\log \left(\left|\alpha_{m}\right|^{2}\right.$ ), is a linear function of $k$ and $\log (\beta)$ (hence also a linear function of $\sigma$ ). For simplicity, we also applied linear interpolation to $\Gamma$. Therefore, we have linearly interpolated these parameters to generate the interpolated CM4 UWB WBAN channel.

Interpolation of CM4 as shown in Fig. 3 is conducted to acquire a $1^{\circ}$ resolution, instead of a $90^{\circ}$ resolution as originally defined in [13] and [15]. The proposed extended channel is determined by a factor of $\left(\frac{b-q}{90}\right)$, where $b$ is the actual body direction and $q \in\left\{0^{\circ}, 90^{\circ}, 180^{\circ}, 270^{\circ}\right\}$.

Let us denote $\Gamma_{b}, k_{b}, \sigma_{b}$ as the exponential decay factor, $K$ factor of NLOS paths, and standard deviation of any single body direction with $1^{\circ}$ resolution, respectively. Further, we denote $\Gamma_{q}, k_{q}$, and $\sigma_{q}$ as the original exponential decay factor, $\mathrm{K}$ factor $k$, and standard deviation
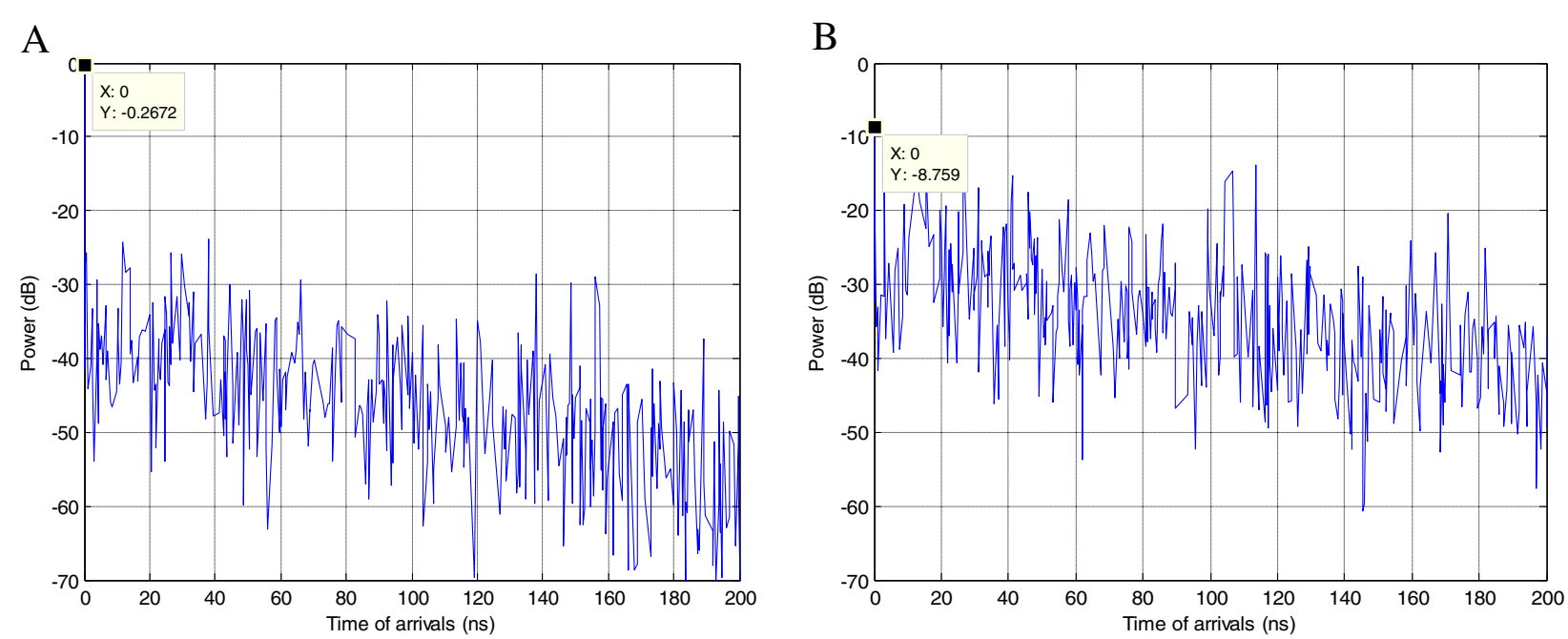

$\mathrm{C}$

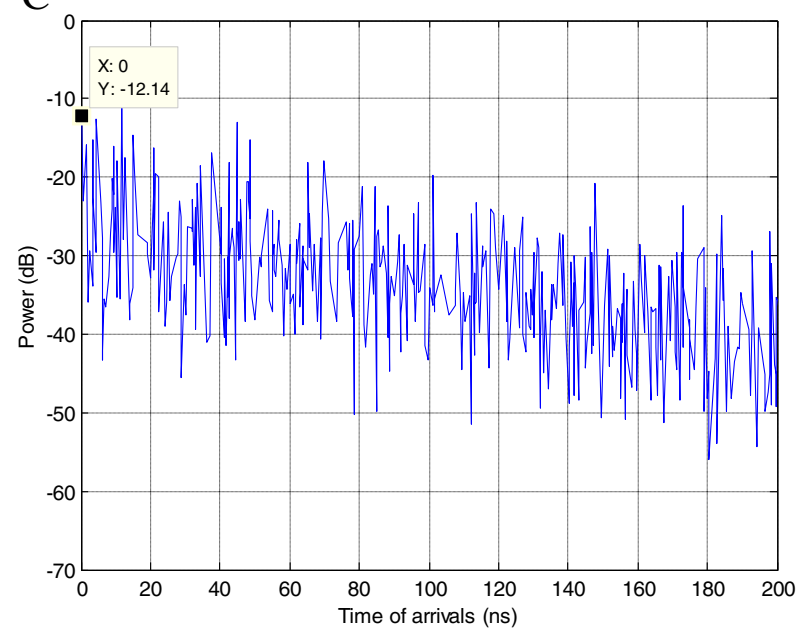

$\mathrm{D}$

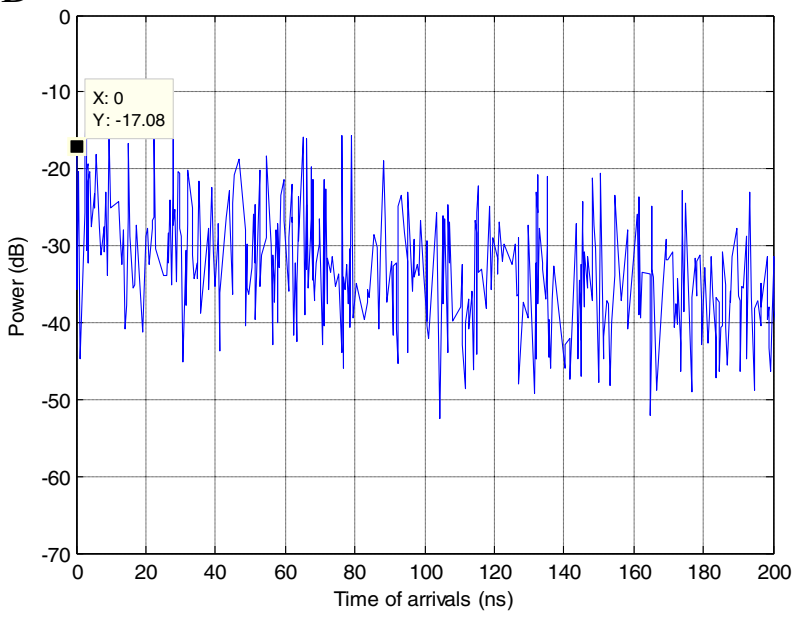

Fig. 2 Power delay profile of the original CM4 at body directions a $0^{\circ}, \mathbf{b} 90^{\circ}, \mathbf{c} 180^{\circ}$, and $\mathbf{d ~} 270^{\circ}$ 


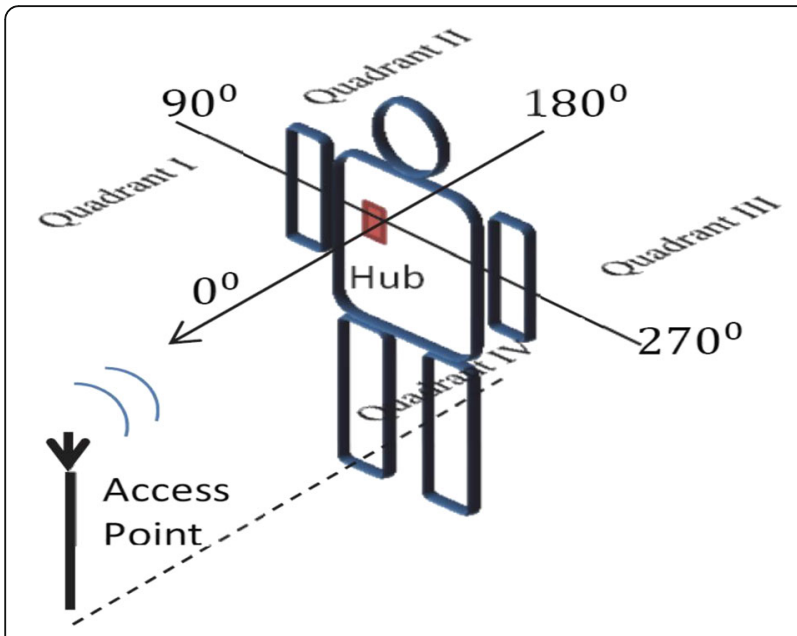

Fig. 3 Proposed interpolated CM4 WBAN channel model

of the $q$ body direction $\left(q \in\left\{0^{\circ}, 90^{\circ}, 180^{\circ}, 270^{\circ}\right\}\right)$ as defined in Table 1 , respectively. Hence, in quadrant I, we have the following relations:

$$
\left.\begin{array}{rl}
\Gamma_{b} & =\Gamma_{0}+\left(\Gamma_{90}-\Gamma_{0}\right) \times\left(\frac{b}{90}\right) \\
k_{b} & =k_{0}+\left(k_{90}-k_{0}\right) \times\left(\frac{b}{90}\right) \\
\sigma_{b} & =\sigma_{0}+\left(\sigma_{90}-\sigma_{0}\right) \times\left(\frac{b}{90}\right)
\end{array}\right\} \text { for } 0^{\mathrm{O}} \leq \mathrm{b} \leq 90^{\circ}
$$

In quadrant II, we have the following interpolation:

$$
\left.\begin{array}{l}
\Gamma_{b}=\Gamma_{90}+\left(\Gamma_{180}-\Gamma_{90}\right) \times\left(\frac{b-90}{90}\right) \\
k_{b}=k_{90}+\left(k_{180}-k_{90}\right) \times\left(\frac{b-90}{90}\right) \\
\sigma_{b}=\sigma_{90}+\left(\sigma_{180}-\sigma_{90}\right) \times\left(\frac{b-90}{90}\right)
\end{array}\right\} \text { for } 90^{\mathrm{O}} \leq \mathrm{b} \leq 180^{\mathrm{O}}
$$

In quadrant III, the following interpolations are applied:

$$
\left.\begin{array}{l}
\Gamma_{b}=\Gamma_{180}+\left(\Gamma_{270}-\Gamma_{180}\right) \times\left(\frac{b-180}{90}\right) \\
k_{b}=k_{180}+\left(k_{270}-k_{180}\right) \times\left(\frac{b-180}{90}\right) \\
\sigma_{b}=\sigma_{180}+\left(\sigma_{270}-\sigma_{180}\right) \times\left(\frac{b-180}{90}\right)
\end{array}\right\} \text { for } 180^{\mathrm{O}} \leq \mathrm{b} \leq 270^{\mathrm{O}}
$$

In quadrant IV, the following equations are applied:

$$
\left.\begin{array}{l}
\Gamma_{b}=\Gamma_{270}+\left(\Gamma_{0}-\Gamma_{270}\right) \times\left(\frac{b-270}{90}\right) \\
k_{b}=k_{270}+\left(k_{0}-k_{270}\right) \times\left(\frac{b-270}{90}\right) \\
\sigma_{b}=\sigma_{270}+\left(\sigma_{0}-\sigma_{270}\right) \times\left(\frac{b-270}{90}\right)
\end{array}\right\} \text { for } 270^{\mathrm{O}} \leq \mathrm{b} \leq 360^{\mathrm{O}}
$$

This interpolation will produce approximate propagation characteristics of the CM4 UWB WBAN channel for every single body direction.

The PDP characteristics of this interpolated CM4 UWB WBAN channel are described in Fig. 4. We intentionally show the PDP of the corresponding body directions of the original CM4 UWB WBAN channel as presented in Fig. 2, plus $1^{\circ}$. This was done so, to show that the interpolated CM4 UWB WBAN channel was able to produce $1^{\circ}$ resolution channel, in addition to the assumption that shifting $1^{\circ}$ angle of direction would not change the channel characteristic significantly. Figure $4 a-d$ resembles the PDP characteristics of the original CM4 UWB WBAN channel (cf. Fig. 2a-d) mentioned above, with minor variations. These facts gave us a solid basis to infer that the interpolated CM4 UWB WBAN channel model was a valid extension of the original CM4 UWB WBAN channel model.

The average number of multipath components $\bar{L}$ generated by this interpolated CM4 UWB WBAN channel model was averaged over $360^{\circ}$ with $1^{\circ}$ step (or in other words, for every single body direction). It was found that $\bar{L}=400.443$. This figure shows that its average number of multipath is similar to that of the original CM4 UWB WBAN channel model $(\bar{L}=400)$. This fact amplifies the conclusion above that the interpolated CM4 UWB WBAN channel model is a valid extension of the original one.

This extended CM4 UWB WBAN channel model is intended to evaluate the comparative performances of the proposed adaptive system with the non-adaptive one employed to the same environment. Thus, it is worth to note that while the particular outcome of this comparative performance investigation achieved by this extended channel might vary should a different channel model be used, the overall idea of the proposed scheme could be still valid for other channel models.

\section{Adaptive STFC MB-OFDM UWB WBAN system 4.1 System model}

Motivated by the fact that the channels behave very differently in the four main directions (c.f. Table 1) resulting in very different performance, we propose here an adaptive WBAN system to improve the overall system performance over all body directions. The main idea is to ensure that the instantaneous system BER never falls 

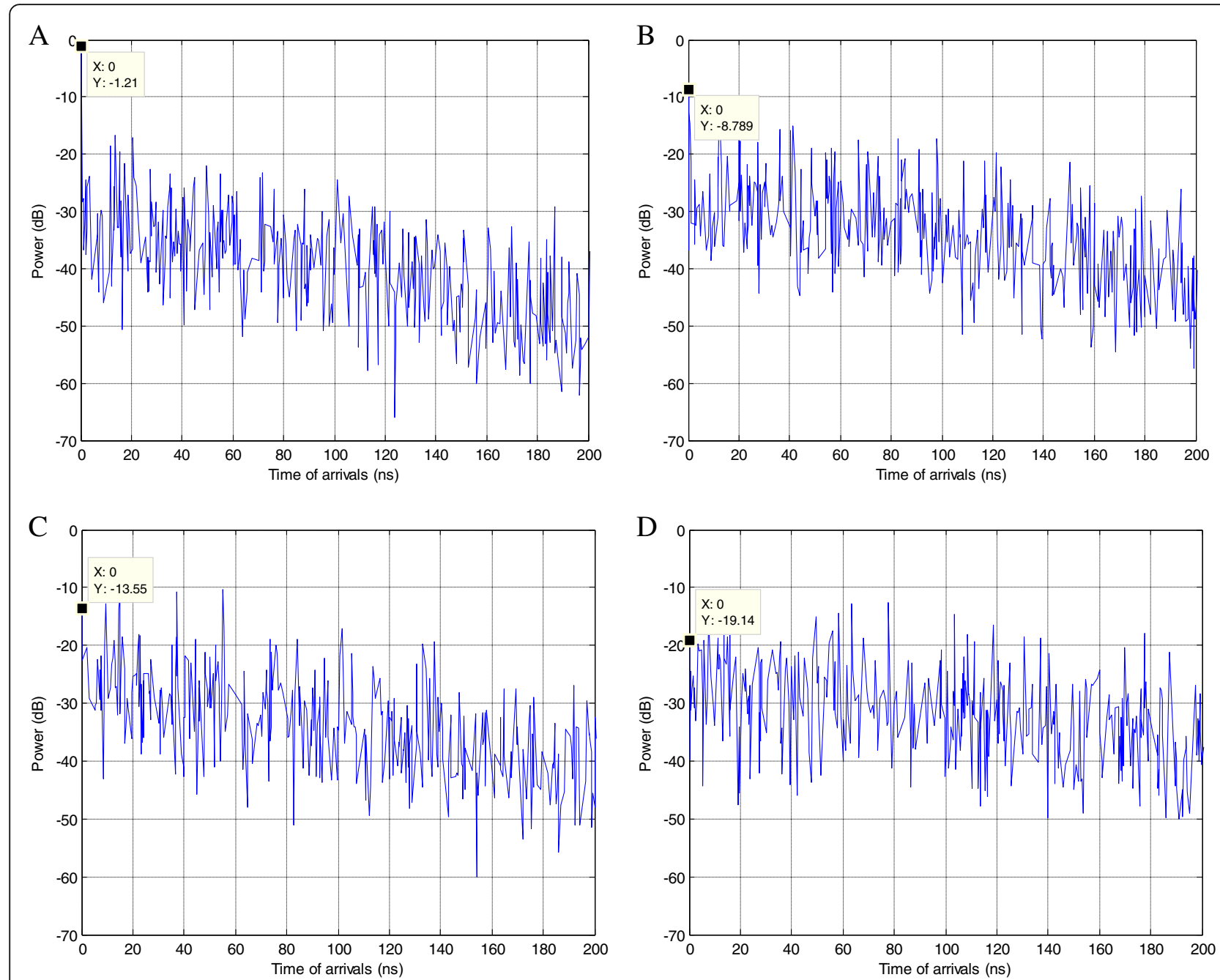

Fig. 4 Power delay profile of the interpolated $C M 4$ at body directions $\mathbf{a} 1^{\circ}, \mathbf{b} 91^{\circ}, \mathbf{c} 181^{\circ}$, and $\mathbf{d} 271^{\circ}$

below a certain threshold by changing the modulation, transmit power, and space-time-frequency coding adaptively to allow the better channel carry more information than the worse one.

The adaptive schemes employed in this system are an evolution from our previously published direction-based adaptive algorithm [28], which are implemented to the original IEEE's CM4 UWB WBAN channel. This channel define only four body directions, i.e., $0^{\circ}, 90^{\circ}, 180^{\circ}$, and $270^{\circ}$. Therefore, we design the adaptive scenario in line with the unique characteristic of this channel model. Initially, we use four adaptive schemes in which two of them are the same due to similar channel conditions (c.f. Table 1). Hence, this requires only three sets of adaptive schemes.

It is widely known that an accurate synchronization is fundamental in ensuring efficient communications and data exchanges between sensors and hub of a WBAN [31]. In particular, dynamic channel changes caused by body movements in WBAN require a precise synchronization on this adaptive system. Therefore, our adaptive WBAN system requires a more stringent synchronization. However, due to the focus of this paper is in the investigation and development of efficient adaptive schemes for WBAN systems, a perfect synchronization is assumed throughout the paper, and we leave the investigation of the synchronization problem for further research.

Figure 5 depicts the proposed high-speed adaptive STFC MB-OFDM UWB WBAN system with $M$ Tx antennas and $N \mathrm{Rx}$ antennas. For the performance comparison purpose, in this paper we only consider $2 \mathrm{I} 1 \mathrm{O}(2$ Tx and $1 \mathrm{Rx}$ antenna), and 2I2O ( $2 \mathrm{Tx}$ and $2 \mathrm{Rx}$ antenna) MIMO configurations. The data stream $d(n)$ is convolutionally encoded and interleaved before being mapped to symbols. The BER estimator measures the channel quality, compares it with the preset thresholds, decides which adaptive scheme Set-l (where $l \in\{1,2,3\}$ ) 


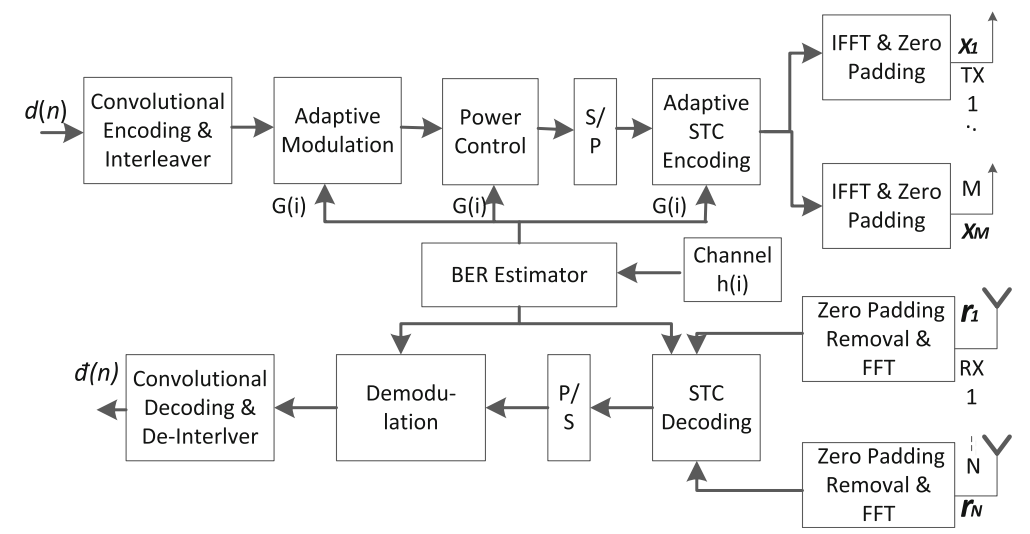

Fig. 5 Proposed high-speed adaptive STFC MB-OFDM UWB WBAN system

to be employed, and feeds this information back to the transmitter in order to adjust its modulation, constellation power, and STFC coding rate.

Selection of the adaptive scheme Set-l is done in a way that the average throughput and total transmitted power, assuming an equal probability of each Set-l to occur, are maintained exactly equal to those in a non-adaptive system for a fair comparison. Here, the non-adaptive system employs QPSK, STFC code rate 1.5, and normalized power 1.0, providing a spectral efficiency of $2 \mathrm{bps} / \mathrm{Hz}$.

Three adaptive schemes are defined as follows: Set-1 is aimed to take advantage of the best channel link by maximizing the throughput, i.e., by employing QPSK modulation, STFC code rate $3 / 2$, and normalized transmission power 1.5, providing a spectral density of $3 \mathrm{bps} / \mathrm{Hz}$; Set-2 is used for the average channel condition, hence employs the same scheme as the non-adaptive system, i.e., by using QPSK, STFC code rate 1, and normalized power 1.0; Set-3 is designed to tackle the worst channel condition by employing lower spectral density BPSK modulation, STFC code rate 1.5 , and normalized power 0.5 , providing a spectral density of $1 \mathrm{bps} / \mathrm{Hz}$.

The adaptive modulation block selects either QPSK or BPSK, while power control block adjusts the power. The stream of modulated symbols is then converted by a serialto-parallel (S/P) block into the symbol blocks (or vectors) $\bar{x}=\left[x_{1}, x_{2}, \ldots, x_{N_{f f t}}\right]^{T}$, where $N_{\text {fft }}$ is the FFT/IFFT size.

The adaptive STFC block creates a space-time code with either full rate or $3 / 2$ rate. The full rate uses the Alamouti code [9] to convert the two consecutive symbol vectors into a STFC block as follows:

$$
\mathbf{X}=\left\{\bar{x}_{t, m}\right\}_{T \times M}=\left[\begin{array}{cc}
\bar{x}_{1} & \bar{x}_{2} \\
-\bar{x}_{2}^{*} & \bar{x}_{1}^{*}
\end{array}\right]
$$

where $\bar{x}_{1}$ and $\bar{x}_{2}$ are symbol vectors transmitted from the first and the second antenna at a given time slot, respectively, and (.)" denotes complex conjugate. For higher code rates, a Sezginer-Sari code [10] or a quasiorthogonal STFC code [11] could be used. Due to lower decoding complexity, here we use the Sezginer-Sari code (3/2-rate STFC), where three symbol vectors are encoded as [10]

$$
\mathbf{X}=\left\{\bar{X}_{t, m}\right\}_{T \times M}=\left[\begin{array}{cc}
a \bar{x}_{1}+\frac{b \bar{x}_{3}}{\sqrt{2}} & -\left(c \bar{x}_{2}^{*}+\frac{d \bar{x}_{3}^{*}}{\sqrt{2}}\right) \\
a \bar{x}_{2}+\frac{b \bar{x}_{3}}{\sqrt{2}} & c \bar{x}_{1}^{*}+\frac{d \bar{x}_{3}^{*}}{\sqrt{2}}
\end{array}\right]
$$

where $a, b, c$, and $d$ are complex-valued design parameters. Here, we use the optimum parameters $a=c=\sqrt{2}$, and $b=d=(1+j \sqrt{7}) / 4$ as determined in [10].

We denote the matrix $\mathbf{X}=\left\{\bar{x}_{t, m}\right\}_{T \times M}$, where $t$ indicates the time slot and $m$ indicates the Tx antenna. Each of symbol vectors in the matrix $\mathbf{X}$ is converted into an $N_{f f t}$-point MB-OFDM symbol by the IFFT block, resulting in the matrix

$$
\mathbf{X}_{\text {OFDM }}=\left\{\bar{x}_{\text {OFDM }, t, m}\right\}_{T \times M}=\left\{\operatorname{IFFT}\left\{\bar{x}_{t, m}\right\}\right\}_{T \times M}
$$

The transmitted matrix is the matrix $\mathbf{X}_{\mathrm{ZP}}$ whose elements are the elements of $\mathbf{X}_{\text {OFDM }}$ appended with a zeropadded suffix (ZPS), denoted as $\bar{x}_{\mathrm{ZP}, t, m}$. This means

$$
\mathbf{X}_{\mathrm{ZP}}=\left\{\bar{X}_{\mathrm{ZP}, t, m}\right\}_{T \times M}
$$

Similar to a cyclic prefix (CP), ZPS is used to mitigate the effects of multipath, hence increase the system robustness against fading. It is also used to create a time window to allow the transmitter and receiver to switch between the different frequencies. CP is created by copying a portion of each OFDM symbol and pastes it to the front of OFDM symbol. As a result, redundancy is introduced into the transmitted signal, hence increases its correlation. This leads to ripples in the average power spectral density (PSD), which, in MB-OFDM systems, 
could be as large as $1.5 \mathrm{~dB}$ [8]. If a ZPS is used, the ripples in the PSD can be minimized, thus the power-back off problem at the transmitter can be avoided, and the system can achieve the maximum possible range.

The channels between $M \mathrm{Tx}$ antennas and $N \mathrm{Rx}$ antennas are defined as the channel matrix $\mathbf{H}$

$$
\mathbf{H}=\left[\begin{array}{ccc}
\bar{h}_{1,1} & \cdots & \bar{h}_{M, 1} \\
\vdots & \ddots & \vdots \\
\bar{h}_{1, N} & \cdots & \bar{h}_{M, N}
\end{array}\right]
$$

where $\bar{h}_{m, n}$ is the channel coefficient vector between the $m$ th Tx antenna, for $m=1,2, \ldots, M$ and the $n$th Rx antenna, $n=1,2, \ldots, N$, containing $L$ multipath. The distribution and parameters of $\bar{h}_{m, n}$ have been shown in Eq. (1) and Table 1.

The received signal at the $n$th $\mathrm{Rx}$ antenna during the $t$ th transmitted OFDM symbol duration is computed as

$$
\bar{r}_{t, n}=\sum_{m=1}^{M}\left(\bar{X}_{\mathrm{ZP}, t, m} \star \bar{h}_{m, n}\right)+\bar{n}_{t, n}
$$

where $(\star)$ denotes the linear convolution, and $\bar{n}_{t, n}$ is the zero mean additive white Gaussian noise vector. The ZPS is removed by an overlap-and-add-operation (OAAO) prior to the FFT operation, thus the received signal can be written as

$$
\bar{r}_{\mathrm{OFDM}, t, n}=\sum_{m=1}^{M} \bar{X}_{\mathrm{OFDM}, t, m} \circledast \bar{h}_{m, n}+\bar{n}_{t, n}
$$

where $(\circledast)$ denotes the circular convolution. After the FFT block, input signals of the STFC decoder are calculated as [8]

$$
\overline{\mathscr{R}}_{t, n}=\sum_{m=1}^{M} \overline{\mathrm{X}}_{t, m} \cdot \overline{\mathscr{H}}_{m, n}+\overline{\mathscr{N}}_{t, n}
$$

where $\quad \overline{\mathscr{R}}_{t, n}=\operatorname{FFT}\left(\bar{r}_{\text {OFDM }, t, n}\right)=\left[\mathscr{R}_{m, n, 1}, \ldots, \mathscr{R}_{m, n, N_{f f t}}\right]^{T}$ is received symbols, $\bar{x}_{t, m}$ is the original modulation symbols, $\overline{\mathscr{H}}_{m, n}=\operatorname{FFT}\left(\bar{h}_{m n}\right)=\left[\hbar_{m, n, 1}, \ldots, \hbar_{m, n, N_{f f t}}\right]^{T}$ is the channel response, $\overline{\mathscr{N}}_{t, n}=\operatorname{FFT}\left(\bar{n}_{t, n}\right)$ is the noise, and $(\bullet)$ denotes the dot (Hadamard) product between vectors. Denote $\mathscr{R}=\left\{\overline{\mathscr{R}}_{t, n}\right\}_{T \times N}$ to be the matrix of the received signals after FFT, $\mathscr{H}=\left\{\overline{\mathscr{H}}_{m, n}\right\}_{M \times N}$ to be the channel response matrix, and $\mathcal{N}=\left\{\overline{\mathscr{N}}_{t, n}\right\}_{T \times N}$ the matrix of noise. Then we can re-write (13) as

$$
\mathscr{R}=\mathbf{X} \circ \mathscr{H}+\mathcal{N}
$$

where $\left(^{\circ}\right)$ denotes the operation which is similar to the normal matrix multiplication, except that each element in $\mathscr{R}$ is determined by (13). Thus, the detected vectors are decided by the following maximum likelihood (ML) rule

$$
\left\{\widetilde{\bar{x}}_{t, m}\right\} \arg \underset{\left\{\bar{x}_{t, m}\right\}}{\min }\|\mathscr{R}-\mathbf{X} \cdot \mathscr{H}\|_{F}^{2}
$$

Since the matrix $\mathbf{X}$ preserves its orthogonality in the similar manner as in a conventional STBC (space-time block code) MIMO system, the STFC MB-OFDM UWB system can also employ a simple linear decoding process. For simplicity, we can omit the time index $t$. Thus, by applying Eqs. (10, 11, 12, 13, and 14) to (15), the ML detection of MB-OFDM symbols employing the Alamouti code in the $2 \mathrm{I} 1 \mathrm{O}$ configuration with M-PSK modulation results in the following decoding metrics:

$$
\begin{aligned}
& \bar{x}_{1}=\underset{\bar{x} \in C^{N_{D}}}{\arg \min }\left\|\left(\overline{\mathscr{H}}_{1}^{*} \cdot \overline{\mathscr{R}}_{1}+\overline{\mathscr{H}}_{2} \bullet \overline{\mathscr{R}}_{2}^{*}\right)-\bar{x}\right\|_{F}^{2} \\
& \bar{x}_{2}=\underset{\bar{x} \in C^{N_{D}}}{\arg \min }\left\|\left(\overline{\mathscr{H}}_{2}^{*} \cdot \overline{\mathscr{R}}_{1}-\overline{\mathscr{H}}_{1} \cdot \overline{\mathscr{R}}_{2}^{*}\right)-\bar{x}\right\|_{F}^{2}
\end{aligned}
$$

where $N_{\mathrm{D}}$ is the number of data subcarriers, and $\mathcal{C}^{N_{\mathrm{D}}}$ denotes the $N_{\mathrm{D}}$-dimensional complex space of the transmitted vector $\bar{x}$. The above decoding metrics are still very complicated. Fortunately, due to the orthogonality, each data point in an MB-OFDM symbol $\left(\bar{x}_{1}\right.$ or $\left.\bar{x}_{2}\right)$ can be decoded separately rather than jointly, thus the decoding process is significantly simplified [8]. Particularly, the decoding metric of each data at the $k$ th subcarrier $\left(k=1, \ldots, N_{D}\right)$ in the MB-OFDM symbols for the $2 \mathrm{I} 1 \mathrm{O}$ configuration are

$$
\begin{aligned}
& \tilde{x}_{1, k}=\arg \min _{x_{1, k} \in \mathcal{C}}\left[\left|\left(\hbar_{1, k}^{*} \mathscr{R}_{1, k}+\hbar_{2, k} \mathscr{R}_{2, k}^{*}\right)-x_{1, k}\right|^{2}\right] \\
& \tilde{x}_{2, k}=\arg \min _{x_{2, k} \in \mathcal{C}}\left[\left|\left(\hbar_{2, k}^{*} \mathscr{R}_{1, k}-\hbar_{1, k} \mathscr{R}_{2, k}^{*}\right)-x_{1, k}\right|^{2}\right]
\end{aligned}
$$

Similarly, for the $2 \mathrm{I} 2 \mathrm{O}$ configuration, the decoding metrics of each data at the $k$ th subcarrier are

$$
\begin{aligned}
& \tilde{x}_{1, k}=\arg \min _{x_{1, k} \in \mathcal{C}}\left[\left|\sum_{n=1}^{2}\left(\hbar_{1, n, k}^{*} \mathscr{R}_{1, n, k}+\hbar_{2, n, k} \mathscr{R}_{2, n, k}^{*}\right)-x_{1, k}\right|^{2}\right] \\
& \tilde{x}_{2, k}=\arg \min _{x_{2, k} \in \mathcal{C}}\left[\left|\sum_{n=1}^{2}\left(\hbar_{2, n, k}^{*} \mathscr{R}_{1, n, k}-\hbar_{1, n, k} \mathscr{R}_{2, n, k}^{*}\right)-x_{2, k}\right|^{2}\right]
\end{aligned}
$$

Generalization of (17) and (18) for the case of $M \mathrm{Tx}$ and $N \mathrm{Rx}$ antennas is straightforward.

For the 3/2-rate STFC, the ML detection of MBOFDM symbols employing the Sezginer-Sari code is slightly different, since this code consists of three symbols $\bar{x}_{1}, \bar{x}_{2}$, and $\bar{x}_{3}$ [10]. In order to reduce the complexity of the receiver, the Sezginer-Sari code detection 
employs two stage detectors. Those are the first stage processor to compute the intermediate signals resulting in the estimate of vector symbol $\bar{x}_{3}$, followed by the predetection of vector symbol $\bar{x}_{1}$ and $\bar{x}_{2}$ given the estimated values of $\bar{x}_{3}$ are known, and the second stage ML estimator to finally decode $\bar{x}_{1}, \bar{x}_{2}$, and $\bar{x}_{3}$ [10]. Here, we briefly summarize the ML detection procedures from [10] as adopted in our proposed system, except that elements of both transmit and receive signal matrix is a vector of MB-OFDM symbols. Consider $2 \mathrm{I} 2 \mathrm{O}$, in which $\mathscr{R}$ is the received signal matrix from the transmit signal matrix $\mathbf{X}$. Denote $\mathbf{Z}$ is the matrix of the intermediate signal whose elements are computed as follows:

$$
\begin{aligned}
& \bar{z}_{1}=\overline{\mathscr{R}}_{1}-\frac{b\left(\overline{\mathscr{H}}_{11}+\overline{\mathscr{H}}_{12}\right) \cdot \bar{x}_{3}}{\sqrt{2}} \\
& \bar{z}_{2}=\overline{\mathscr{R}}_{2}-\frac{d\left(\overline{\mathscr{H}}_{11}+\overline{\mathscr{H}}_{12}\right) \cdot \bar{x}_{3}^{*}}{\sqrt{2}} \\
& \bar{z}_{3}=\overline{\mathscr{R}}_{3}-\frac{b\left(\overline{\mathscr{H}}_{21}+\overline{\mathscr{H}}_{22}\right) \cdot \bar{x}_{3}}{\sqrt{2}} \\
& \bar{z}_{4}=\overline{\mathscr{R}}_{4}-\frac{d\left(\overline{\mathscr{H}}_{21}+\overline{\mathscr{H}}_{22}\right) \cdot \bar{x}_{3}^{*}}{\sqrt{2}}
\end{aligned}
$$

for a given value of $\bar{x}_{3}$. By mathematical manipulation as analyzed in Eq. (15a) to Eq. (16d) of [10], we use this intermediate signal to calculate the estimate of $\widetilde{\bar{x}}_{1}$ and $\widetilde{\bar{x}}_{2}$ conditional on $\widetilde{\bar{x}}_{3}$ as follows:

$$
\begin{aligned}
\tilde{\bar{x}}_{1} & =\frac{\left(\overline{\mathscr{H}}_{11}^{*} \cdot \bar{z}_{1}+\overline{\mathscr{H}}_{21}^{*} \cdot \bar{z}_{3}\right)}{a}+\frac{\left(\overline{\mathscr{H}}_{12} \bar{z}_{2}^{*}+\overline{\mathscr{H}}_{22} \cdot \bar{z}_{4}^{*}\right)}{c^{*}} \\
& =\left(\left|\overline{\mathscr{H}}_{11}\right|^{2}+\left|\overline{\mathscr{H}}_{12}\right|^{2}+\left|\overline{\mathscr{H}}_{21}\right|^{2}+\left|\overline{\mathscr{H}}_{22}\right|^{2}\right) \cdot \bar{x}_{1}
\end{aligned}
$$

and

$$
\begin{aligned}
\tilde{\bar{x}}_{2} & =\frac{\left(\overline{\mathscr{H}}_{12}^{*} \cdot \bar{z}_{1}+\overline{\mathscr{H}}_{22}^{*} \cdot \bar{z}_{3}\right)}{a}-\frac{\left(\overline{\mathscr{H}}_{11} \cdot \bar{z}_{2}^{*}+\overline{\mathscr{H}}_{21} \bar{z}_{4}^{*}\right)}{c^{*}} \\
& =\left(\left|\overline{\mathscr{H}}_{11}\right|^{2}+\left|\overline{\mathscr{H}}_{12}\right|^{2}+\left|\overline{\mathscr{H}}_{21}\right|^{2}+\left|\overline{\mathscr{H}}_{22}\right|^{2}\right) \cdot \bar{x}_{2}
\end{aligned}
$$

As has been mentioned previously, each data point in an MB-OFDM symbol can be decoded separately rather than jointly [8]. By applying each element of vector $\widetilde{\bar{x}}_{1}$ and $\widetilde{\bar{x}}_{2}$ to a threshold detector, each data symbol at the $k$ th subcarrier, for $k=1, \ldots, N_{D}$ can be decoded as follows:

$$
\tilde{x}_{1, k}=\left(\left|\hbar_{11}\right|^{2}+\left|\hbar_{12}\right|^{2}+\left|\hbar_{21}\right|^{2}+\left|\hbar_{22}\right|^{2}\right) x_{1, k}
$$

and

$$
\tilde{x}_{2, k}=\left(\left|\hbar_{11}\right|^{2}+\left|\hbar_{12}\right|^{2}+\left|\hbar_{21}\right|^{2}+\left|\hbar_{22}\right|^{2}\right) x_{2, k}
$$

At this point, we have estimated $\tilde{x}_{1, k}$ and $\tilde{x}_{2, k}$ given symbol $x_{3, k}$ is known. Now, to estimate the symbol $x_{3, k}$ by ML detector, we need to compute the Euclidean distance for $\left(\tilde{x}_{1, k}, \tilde{x}_{2, k}, x_{3, k}\right)$ to get $\tilde{x}_{3, k}$. By this mean, it only requires a linear ML detector [10]. Readers may refer to [10] for a detailed analysis of the Sezginer-Sari code detection.

\subsection{Transmission model}

The proposed transmission model is described in Fig. 6. For clarity, it is assumed that a person wearing WBAN devices makes an anti-clockwise angular movement with respect to a fixed external transceiver. Different angles of the body direction experience dissimilar radio propagation characteristics, leading to different fading channels [15]. During each frame transmission, the receiver measures the signal quality, i.e., BER, in the portion $f$ of the frame, $(0<f<1)$, where Set-2 is selected as the default modulation and STFC coding scheme. The measured BER is not fed back directly to the transmitter. Instead, it will be compared to the preset thresholds (discussed in the next section) to select one out of three possible sets of adaptive schemes, denoted as Set-l, for $l \in\{1,2,3\}$. Hence, only two bits information, as shown in Table 2, are required to be fed back to the transmitter to indicate which set of adaptive schemes should be employed by the transmitter for the remaining $(1-f)$ portion of that frame. Note that this Set-l is known to the receiver. After completion of a frame transmission, the first portion $f$ of the next frame will utilize Set-2 again, followed by the same procedure as above.

From a practical point of view, this simple transmission model and simple adaptive scheme are favorable, particularly in coping with the channel changing due to body movement. In addition, time delay caused by the channel BER measurement, feedback, and frame transmission is considered negligible since the distance between TX and RX is around 2-5 $\mathrm{m}$ in body-to-external WBAN links. Thus, these three factors, namely, simple

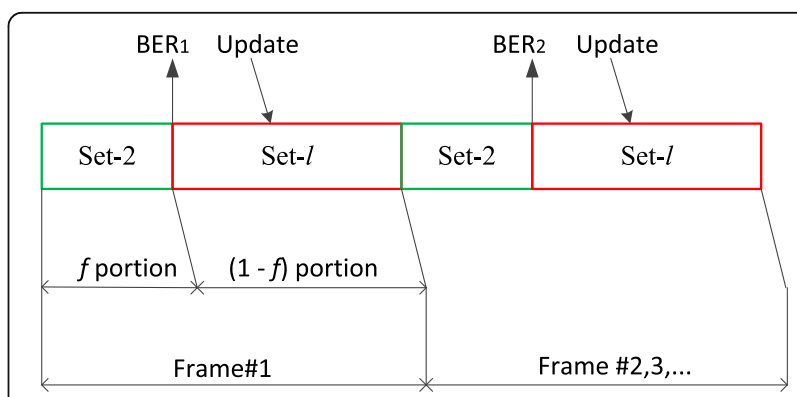

Fig. 6 Adaptive frame transmission model 
Table 2 2-bit information and the corresponding adaptive sets

\begin{tabular}{ll}
\hline 2-bit feedback signal & Corresponding adaptive set \\
\hline 00 & Set-1 \\
01 & Set-2 \\
10 & Set-3 \\
11 & Reserved for future usage \\
\hline
\end{tabular}

feedback mechanism, the receiver's prior knowledge of the Set-l, and very low delay, practically allow this adaptive system to quickly adapt to the dynamic channel changes due to the body movement.

\subsection{Adaptive algorithm}

The adaptive scheme is controlled by the measurement of the signal quality, i.e., BER, at Rx. The measured BER will then be compared to the BER thresholds. Two BER thresholds are defined in this paper, namely, upper and lower thresholds that determine the selection of one of three possible schemes denoted as Set-l. The upper and lower thresholds are used and based on the non-adaptive average BER performance as the benchmark.

Two methods are proposed to define the preset thresholds that trigger the change of adaptive schemes, namely, linear and non-linear BER thresholds. The linear approach defines the lower and upper thresholds, which are below and above the non-adaptive average BER performance, respectively, and always have a constant distance from this non-adaptive BER curve within the whole range of the considered signal-to-noise ratio (SNR), based on (27) and (28)

$$
\begin{aligned}
& \mathrm{BER}_{L}^{(g)}=\mathrm{BER}_{\mathrm{NA}}^{(g)}-k \times \mathrm{BER}_{\mathrm{NA}}^{(g)} \\
& \mathrm{BER}_{U}^{(g)}=\mathrm{BER}_{\mathrm{NA}}^{(g)}+k \times \mathrm{BER}_{\mathrm{NA}}^{(g)}
\end{aligned}
$$

where $\mathrm{BER}_{L}^{(g)}$ is the lower threshold, $\mathrm{BER}_{U}^{(g)}$ the upper threshold, and $\mathrm{BER}_{\mathrm{NA}}^{(g)}$ the non-adaptive average BER performance for the $g$ th SNR value; $k$ is a constant, whose value will be optimized later in this paper.

The non-linear BER thresholds define a smaller deviation w.r.t. to the non-adaptive average BER performance at low SNRs, and the deviation is non-linearly increased when the SNR increases. This approach aims to allow higher probability of Set-1 to occur, resulting in a higher throughput, when the channel condition is better (higher SNR). The lower and upper non-linear thresholds are defined as follows:

$$
\begin{aligned}
& \mathrm{BER}_{L, n l}^{(g)}=\mathrm{BER}_{\mathrm{NA}}^{(g)}-k \times \mathrm{BER}_{\mathrm{NA}}^{(g)} \times(1+p)^{g} \\
& \mathrm{BER}_{U, n l}^{(g)}=\mathrm{BER}_{\mathrm{NA}}^{(g)}+k \times \mathrm{BER}_{\mathrm{NA}}^{(g)} \times(1+p)^{g}
\end{aligned}
$$

where $p$ is a constant. The subscript $n l$ denotes the non-linear threshold approach.
The proposed algorithm for each frame transmission is summarized in Table 3.

The decoding process of Set-1 (QPSK and the 3/2-rate STFC code) has more complexity compared to other sets, since the decoder firstly has to determine $\tilde{x}_{3}$ from four possible symbols in QPSK, prior to decoding $\tilde{x}_{1}$ and $\tilde{x}_{2}$ symbols. Then, $\tilde{x}_{1}$ and $\tilde{x}_{2}$ are decoded independently, given $\tilde{x}_{3}$ is known, for every QPSK constellation. Hence, if $P$ is the constellation size of the modulator, then the ML decoding complexity is the decoding complexity of $\tilde{x}_{3}$, which is in the order of $P$, added with the complexity of two independent decoding processes of $\tilde{x}_{1}$ and $\tilde{x}_{2}$ given $\tilde{x}_{3}$ is known, which is equal to $2 P$. Thus the overall decoding complexity of Set-1 is in the order of 3P. Meanwhile, in the worse links, the complexity is a little bit reduced. Set-2 uses a QPSK modulator with the full-rate Alamouti code as the STFC in which symbols $\tilde{x}_{1}$ and $\tilde{x}_{2}$ are decoded independently for every constellation result in $2 P$-decoding complexity. Set-3 uses the Alamouti code in which $\tilde{x}_{1}$ and $\tilde{x}_{2}$ symbols are also decoded independently for every BPSK constellation point. Therefore the ML decoding complexity in Set-3 is $P$. Thus, it can be concluded that the ML decoding complexity is in the order of $P$ or $O(P)$. It is obvious that the

Table 3 BER-based adaptive WBAN algorithm

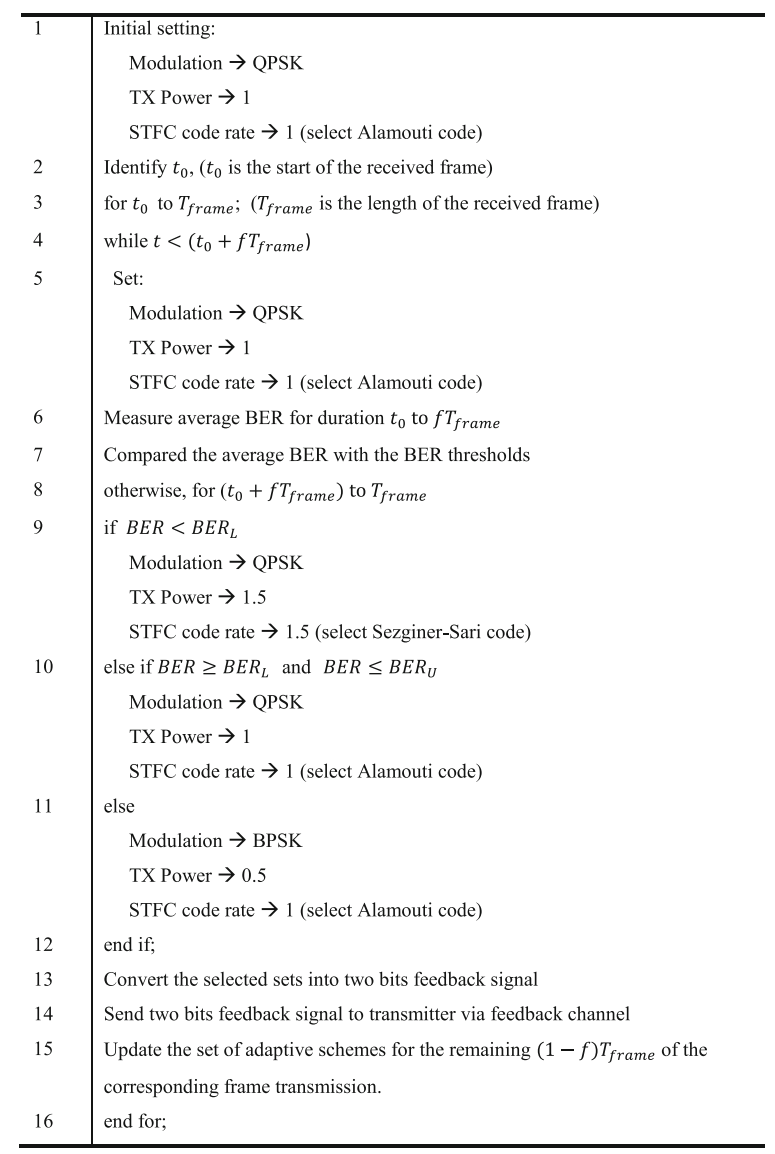


complexity of this adaptive system only linearly increases with respect to the number of signal constellations, thanks to relatively simple decoding processes.

\section{Optimization of $\boldsymbol{f}$ and $\boldsymbol{k}$ parameters}

Performance of the adaptive system depends on the selected Set-l on a frame-to-frame basis. During the $q$ th frame transmission, where $q=0,1,2, \ldots, Q-1$, and $Q$ is the number of frames, the measurement of BER and adaptation occur during the $f$ portion of the frame as described above. Hence, the overall BER of the system is affected by the selected value of the $f$ factor. At the same time, BER is also affected by the selection of $S e t-l$, which is done by comparing the measured BER of the current received frame with the thresholds, which are, in turn defined by the $k$ factor. Hence the system performance depends on both $f$ and $k$.

In this section, $f$ and $k$ will be optimized. However, it should be noted that optimization of $f$ factor is done in the initial stage to find the optimized $f$ value, then use it as a fixed value to design the frame structure. We only consider the linear threshold approach because it is simpler and more importantly it produces better BER performance compared to the non-linear threshold approach, as explained in the next section. Referring to Fig. 4 , it is clear that $0<f<1$. The $f$ factor must also be a fraction $(0<k<1)$ and the following conditions apply, (i) $k \neq 0$. If $k=0$, the upper threshold will be equal to the lower threshold resulting in no threshold. (ii) $k \neq 1$. If $k=1$, the upper threshold will be equal to the benchmark value; hence, the adaptive system will deliver no better performance compared to the non-adaptive system.

For simplicity, but without loss of generality, we consider a set of limited discrete values of $f$ and $k$ as detailed in (31) and (32). Note that $f$ and $k$ are independent from each other.

$$
\begin{aligned}
f_{i} & =0.1 i, i=1,2, \ldots, 9 \\
k_{j} & =0.1 \mathrm{j}, j=1,2, \ldots, 9
\end{aligned}
$$

We denote a binary variable $u_{i}^{q}$ where $u_{i}^{q}=1$ if a certain portion of the frame $f_{i}$ is selected to measure the BER, and $u_{i}^{q}=0$ otherwise. We define a binary variable $v_{j}^{q}$ where $v_{j}^{q}=1$ if a certain value of $k_{j}$ is selected to determine the upper and lower BER thresholds as mentioned in (27) and (28), and $v_{j}^{q}=0$ otherwise.

We also denote $z_{i}^{q}$ and $w_{j}^{q}$ to be the costs associated with the selected $f_{i}$ and $k_{j}$, respectively. The costs here mean the BER performance. Since the maximum likelihood decoding is used, the costs could be expressed as arg $\min _{\left\{\bar{x}_{t, m}\right\}}\|\mathscr{R}-\mathbf{X} \bullet \mathscr{H}\|_{F}^{2}$.

Our objective is to optimize the overall average BER performance of all $Q$ frames by finding an optimized $f$ and $k$ factors, assuming that frame transmission in every direction of the body is equally probable. Hence, the total cost function is defined by

$$
\text { cost }=\sum_{q=0}^{Q-1} \sum_{i=1}^{9} \sum_{j=1}^{9}\left(z_{i}^{q} \cdot u_{i}^{q}+w_{j}^{q} \cdot v_{j}^{q}\right)
$$

The first term of (33) represents the average BER performance associated with a given value of $f$, provided that $k$ is randomly chosen. The second term of (33) is likewise for a given value $k$, provided $f$ is randomly selected.

Note that given the optimized values of $f$ is chosen at the initial stage as previously mentioned, only a $k$ value is chosen during each frame transmission. This is equivalent to selecting a single optimized value of $u_{i}^{q}$ and $v_{j}^{q}$ from their possible values. Thus, the constraints are determined by

$$
\begin{aligned}
& \sum_{i=1}^{9} u_{i}^{q}=1, \forall i=1,2, \ldots, 9 \\
& \sum_{j=1}^{9} v_{j}^{q}=1, \forall j=1,2, \ldots, 9 \\
& u_{i}^{q} \geq 0, v_{j}^{q} \geq 0
\end{aligned}
$$

It is clear that the above optimization problem is equivalent to minimization of the total cost function (33) subject to the constraints (34)-(36).

Denote vector $\quad \mathbf{u}=\left(u_{1}^{0}, ., u_{9}^{0}, u_{1}^{1}, ., u_{9}^{1}, ., u_{10-1}, ., u_{90-1}\right) \quad T \in$ $\{0,1\}^{9 Q \times 1}, \mathbf{v}=\left(v_{1}^{0}, . ., v_{9}^{0}, v_{1}^{1}, . ., v_{9}^{1}, . ., v_{1^{Q-1}}, . ., v_{9^{Q-1}}\right)^{T} \in\{0,1\}^{9 Q \times 1}$, $\mathbf{z}=\left(z_{1}^{0}, . ., z_{9}^{0}, z_{1}^{1}, . ., z_{9}^{1}, . ., z_{1}^{Q-1}, . ., z_{90-1}\right)^{T} \in \mathbb{R}^{9 Q \times 1}, \mathbf{w}=\left(w_{1}^{0}, . ., w_{9}^{0}\right.$, $\left.w_{1}^{1}, . ., w_{9}^{1}, . ., w_{1 \mathrm{Q}^{\mathrm{-}}}, . ., w_{9 \mathrm{Q}-1}\right)^{T} \in \mathbb{R}^{9 \mathrm{Q} \times 1}$, where $\mathbb{R}$ denotes a field of non-negative real numbers. Then, the cost function (33) can be expressed mathematically as

$$
\text { cost }=\mathbf{z}^{T} \mathbf{u}+\mathbf{w}^{T} \mathbf{v}
$$

The first constraint (22) can be written as

$$
\mathbf{A u}=1_{Q}
$$

where $\mathbf{A}=\mathbf{I}_{Q} \otimes 1_{9}^{T} \in\{0,1\}^{Q \times 9 Q}, \mathbf{I}_{Q}$ is a $Q \times Q$ identity matrix, $\otimes$ denotes the Kronecker product, and $1_{9}$ is an all one $9 \times 1$ vector. Similarly, (23) becomes

$$
\mathbf{A v}=1_{Q}
$$

Thus the optimization problem can be formulated as

$$
\begin{aligned}
& \min _{u, v \in\{1,0\}\}^{Q \times 1}} \mathbf{z}^{T} \mathbf{u}+\mathbf{w}^{T} \mathbf{v} \\
& \text { s.t. } \mathbf{A u}=1_{Q}, \mathbf{A v}=1_{Q}
\end{aligned}
$$

Clearly, (40) is a standard linear optimization problem with binary elements since the vectors $\mathbf{u}$ and $\mathbf{v}$ only consist of 0 and 1 entries [32,33]. To solve the optimization 
problem, we exploit the unique characteristic of the matrix $\mathbf{A}$ by using the total unimodularity concept. A is totally unimodular (TUM) if the determinant of every square submatrix of $\mathbf{A}$ has a value of $-1,0$, or 1 [33]. It can be shown that the constraint matrix $\mathbf{A}$ is indeed TUM. The proof is analogous to Eq. (18) in [34].

From the integer optimization theory, if $\mathbf{A}$ is TUM and both $\mathbf{u}$ and $\mathbf{v}$ are integer vectors, the extreme points of possible solutions (polyhedron) are integral. Hence, the optimization problem can be solved using linear programming, for instance, using a well-known simplex or interior point method [33]. By using this procedure, we get optimum $f$ factor of 0.3 . This means that the measurement of instantaneous BER should take place at the first $30 \%$ of the frame and update the adaptive scheme for the rest of the frame transmission thereon. An optimum $k$ factor is also obtained and equal to 0.6, which means that the distance between lower and upper thresholds is $3 \mathrm{~dB}$.

\section{Simulation results}

6.1 Proofs of the validity of the interpolated CM4 UWB WBAN channel model

In order to prove further the validity of the interpolated CM4 UWB WBAN channel model, simulations were conducted in MATLAB. The body direction model can be seen in Fig. 3. The simulation employs the same scheme for all quadrant, i.e., by using QPSK modulation 2I1O (2 $\mathrm{Tx}$ and $1 \mathrm{Rx}$ antenna) MB-OFDM UWB WBAN. Body directions in each quadrant are randomly chosen. The evaluation uses a BER metric to comparatively assess the change of the channel behavior associated with the angle of body direction.

As mentioned previously, according to the original CM4 UWB WBAN channel model, the best link occur in $0^{\circ}$ (line of sight, LOS), then $90^{\circ}, 180^{\circ}$, and the worst link is $270^{\circ}$. Intuitively, the channel fading will sequentially degrade when the angle of body direction gradually increase from $0^{\circ}$ to $90^{\circ}$ (quadrant I), from $90^{\circ}$ to $180^{\circ}$ (quadrant II), from $180^{\circ}$ to $270^{\circ}$ (quadrant III), and from $0^{\circ}$ to $270^{\circ}$ (quadrant IV). This sequential degradation of the fading channel should be consistent with any step of the gradual increase of the body direction angle. The simulation results prove that all conditions and hypothesis above are fulfilled. For example, Fig. 7 and Fig. 8 show the sequential performance degradation due to changing channel fading for random body direction in quadrants I and III, respectively. This is also true for $1^{\circ}$ step of gradual change of the angle of body direction. The same results are also consistently produced in the other quadrants. Thus, we conclude that the extended channel model is valid for any degree with $1^{\circ}$ resolution.

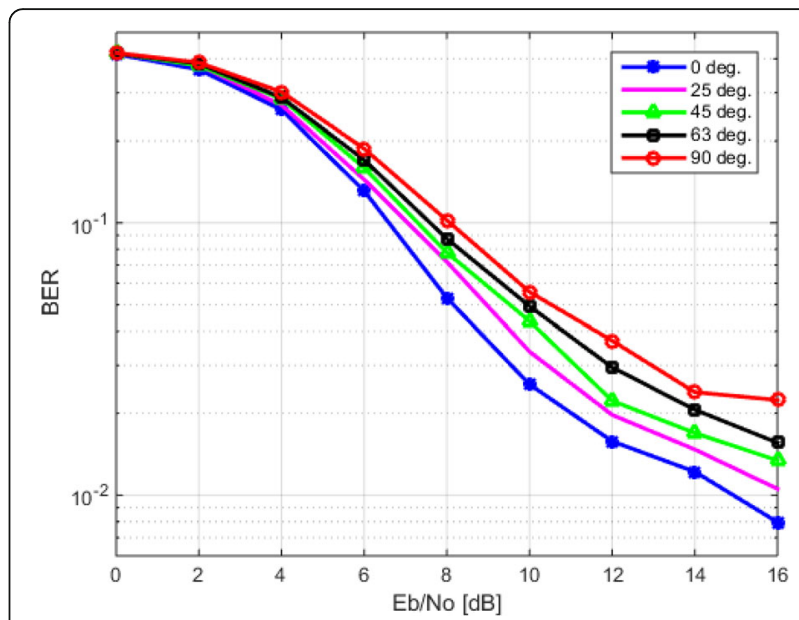

Fig. 7 Performance degradation due to changing channel fading for random body direction in quadrant I

\subsection{Performance evaluation}

This section compares system BER performances of the non-adaptive system, the adaptive system, and optimized adaptive system. It is assumed that the transmitter completes the transmission of one data frame after every $15^{\circ}$ angular movement of the body, and a perfect channel state estimation is available at the receiver. Channel coefficients are assumed to be constant during each STFC block, but random between consecutive STFC blocks [35]. The channel realizations are created based on our extended CM4 model. MB-OFDM UWB employs a bandwidth of $528 \mathrm{MHz}$ and other parameters as listed in Table 4 . The STFCs are implemented in the $2 \times 1$ and $2 \times 2$ MIMO configurations. For illustration, before optimizing $f$ and $k$ factors, we select $f$ equal to $0.25, p$ equal to 0.175 , and $k$ equal to 0.5 and 0.4 for linear and nonlinear threshold approaches, respectively. As for optimization results in Fig. 11, we use the optimum $f$

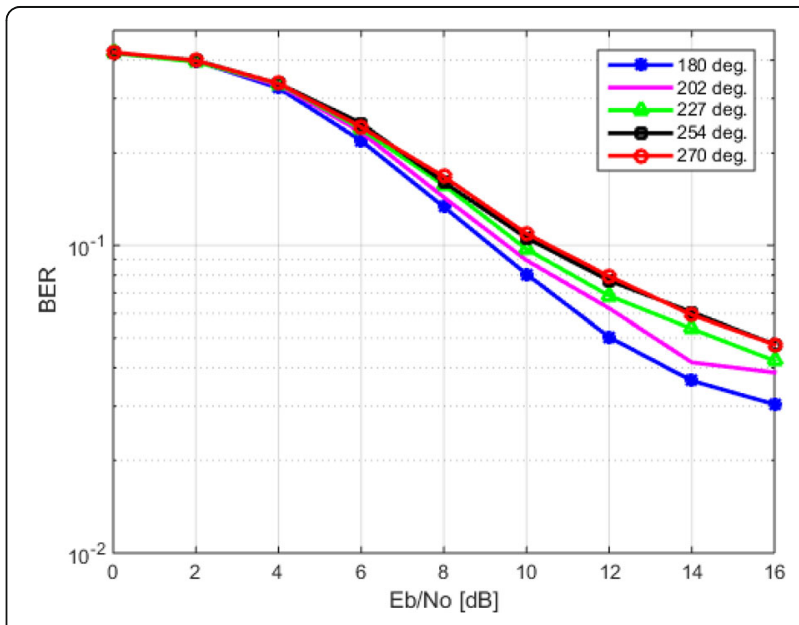

Fig. 8 Performance degradation due to changing channel fading for random body direction in quadrant III 
Table 4 Simulation parameters

\begin{tabular}{ll}
\hline Parameters & Values \\
\hline FFT and IFFT size $N_{\text {fft }}$ & 128 \\
Number of ZPS $N_{\text {ZPS }}$ & 37 \\
Convolutional coder $(K=7)$ rate & $1 / 2$ \\
Conv. decoder and mode & Viterbi, hard \\
\hline
\end{tabular}

and $k$ values as previously mentioned in Section 5, i.e., $f=0.3$ and $k=0.6$.

Figure 9 shows the average performance of nonoptimized adaptive system for both (a) $2 \mathrm{I} 1 \mathrm{O}$ and (b) 2I2O MIMO configurations, compared to the average non-adaptive system performance. Clearly, the proposed adaptive system with both BER threshold approaches performs better than the non-adaptive system. In the 2I1O configuration, the improvement in the order of 1$2 \mathrm{~dB}$ is achieved by non-linear BER threshold and of $1-$ $4 \mathrm{~dB}$ by the linear BER threshold within the low to medium SNR range where most of the WBAN systems are expected to operate. These gains show a significant improvement for WBAN applications. This proposed adaptive system could potentially save the $\mathrm{Tx} / \mathrm{Rx}$ power at a level of $20-60 \%$, compared to the non-adaptive system. With this level of power reduction, the adaptive STFC MB-OFDM UWB system could be an energy-efficient alternative for high data rate WBAN applications. It is important to highlight the capability of this adaptive WBAN in offering a much higher data rate, i.e., $1 \mathrm{Gbps}$, compared to impulse radio (IR) UWB-based WBAN systems whose maximum data rate is $15.6 \mathrm{Mbps}$ [3].

From Fig. 9, one can also see that the adaptive system in a $2 \mathrm{I} 2 \mathrm{O}$ configuration provides a slightly less improvement over the non-adaptive one, compared to the $2 \mathrm{I} 1 \mathrm{O}$ configuration. The gain is about $1-2 \mathrm{~dB}$ or $20-37 \%$ transmission power saving for the linear BER threshold. The non-linear BER threshold performs slightly worse than the linear BER threshold in the whole SNR region. The improvement is smaller in the $2 \mathrm{I} 2 \mathrm{O}$ configuration since the system already has a large diversity, so even the non-adaptive WBAN system could take advantage of it. Nonetheless, if we compare the error performances of the $2 \mathrm{I} 1 \mathrm{O}$ and $2 \mathrm{I} 2 \mathrm{O}$ configurations, it is intuitive that the application of the proposed adaptive schemes in the 2I2O configuration provides a much better link quality up to BER of $10^{-4}$ that is suitable for more stringent QoS applications.

The average normalized throughput taken from 24 different body directions are shown in Fig. 10. Delays of the channel quality measurement, feedback, and frame transmission are considered negligible since the distance between $\mathrm{Tx}$ and $\mathrm{Rx}$ is around $2-5 \mathrm{~m}$ in body-to-external WBAN links. Hence, the throughput is not affected by those delays. At low SNRs, Set-3 with a lower spectral efficiency is most likely employed, resulting in a lower throughput for both linear and non-linear BER thresholds. At high SNR values, higher spectral efficiency adaptive schemes, i.e., Set-1 and Set-2, are employed more frequently, leading to a higher throughput approaching the nonadaptive throughput of $2 \mathrm{bps} / \mathrm{Hz}$. However, the gap between the non-adaptive and adaptive throughputs is still significant, even at the high SNR.

From Fig. 10, one can realize that, within the $E_{\mathrm{b}} / N_{\mathrm{o}}$ range [0-6] $\mathrm{dB}$ for $2 \mathrm{I} 1 \mathrm{O}$ and $[0-1] \mathrm{dB}$ for $2 \mathrm{I} 2 \mathrm{O}$, the linear BER threshold approach tends to have higher throughput compared to that of non-linear BER threshold. Thus, its BER performance within these ranges is slightly worse than that of the non-linear BER threshold approach. This slight performance difference between
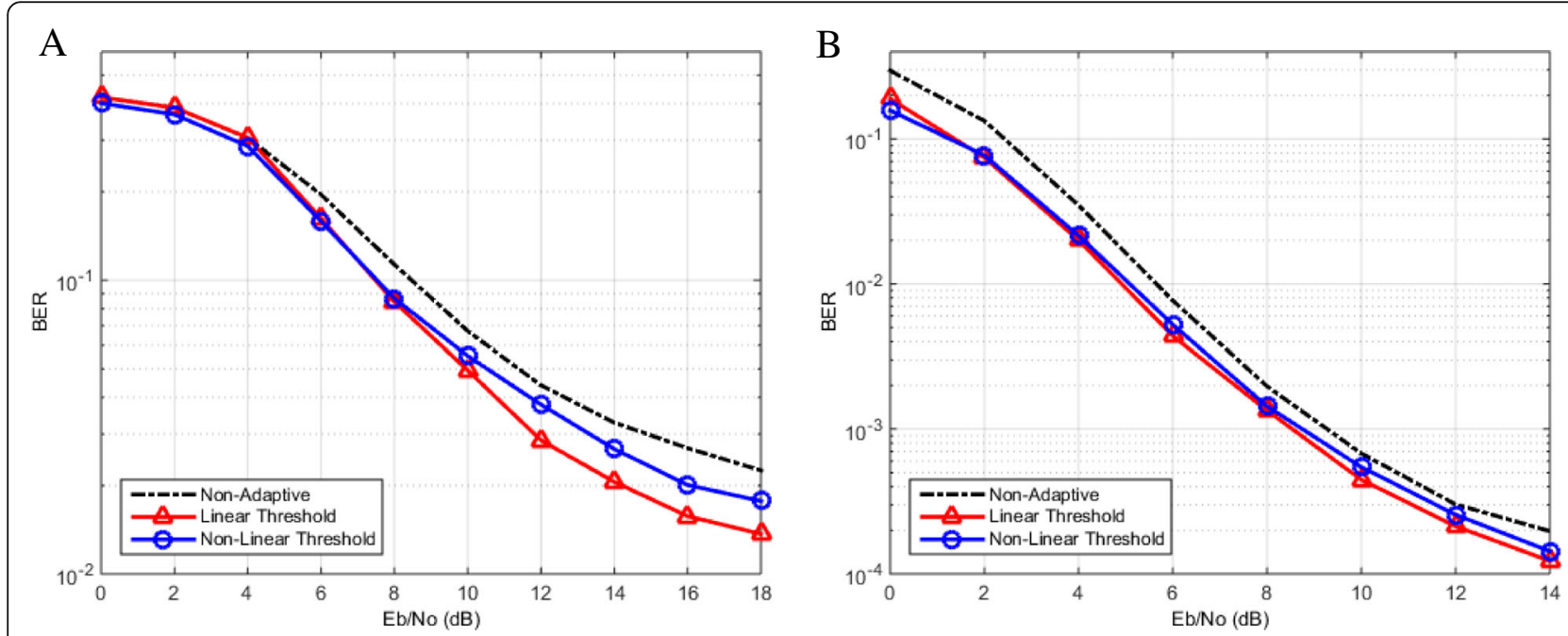

Fig. 9 Performances of non-optimized adaptive WBAN in a 2110 and $\mathbf{b} 2120$ 


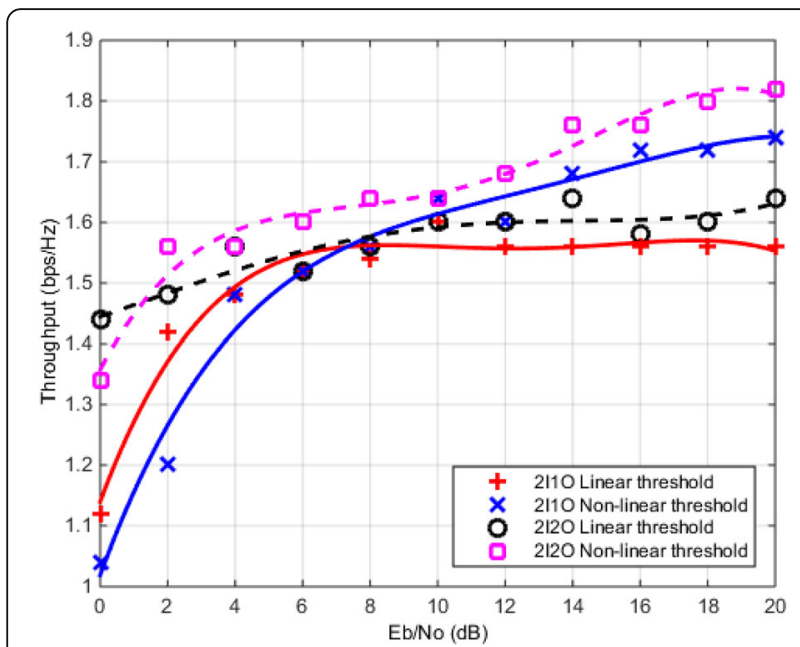

Fig. 10 Throughput of non-optimized adaptive scheme

the two approaches can be seen clearly in Fig. 9. These are due to the fact that at low SNRs, the distance between the upper and lower threshold to the benchmark non-adaptive performance curve is smaller in the nonlinear threshold, compared to that in the linear approach. Hence, at low SNR the adaptive systems with non-linear threshold utilize more frequently adaptive scheme Set-3 rather than other sets, resulting in aforementioned conditions.

Figure 11 presents the additional performance improvement achieved by the optimization of $f$ and $k$ parameters. Particularly, Fig. 11 compares the performances of the optimized and non-optimized adaptive systems in the $2 \mathrm{I} 1 \mathrm{O}$ (a), and in the $2 \mathrm{I} 2 \mathrm{O}$ (b). In the medium to high SNR range, the non-optimized adaptive $2 \mathrm{I} 1 \mathrm{O}$ system provides a $1-4-\mathrm{dB}$ gain over the non-adaptive counterpart. The optimized adaptive system enhances the performance further by 1-2 $\mathrm{dB}$ in the whole SNR range. Meanwhile, the nonoptimized adaptive $2 \mathrm{I} 2 \mathrm{O}$ WBAN provides a near constant $1-2-\mathrm{dB}$ gain in the whole range of SNR, compared to the non-adaptive WBAN. Again, optimizing the $f$ and $k$ parameters provides an additional 1-2-dB improvement.

Therefore, in these two MIMO configurations, the optimized adaptive WBAN results in a gain of up to $2 \mathrm{~dB}$ over the non-optimized adaptive WBAN, and up to $6 \mathrm{~dB}$ over the non-adaptive WBAN. Translating to the power saving, the optimized adaptive approach provides a power consumption reduction by up to 37 and $75 \%$, compared to the two counterparts. Overall, the schemes achieve the aim, i.e., gaining better BER performance for high data rate WBAN applications with a reasonably high throughput.

\section{Conclusions}

This paper proposes a novel optimized BER-based adaptive STFC MB-OFDM UWB WBAN system. It is designed to improve the average bit error performance for high data rate applications while maintaining a reasonably high throughput in body-to-external links of a WBAN. The proposed adaptive WBAN comprises two novel components, namely, the BER estimation-based adaptive scheme, and the optimization of its adaptive parameters, i.e., $f$ and $k$ factors, in order to obtain an extra improvement, hence enhancing the performance further. The adaptive system is evaluated over the interpolated CM4 UWB WBAN channel.

The proposed adaptive WBAN system provides a significant gain of up to $4 \mathrm{~dB}$, compared to the nonadaptive one. By the optimization of its adaptive parameters ( $f$ and $k$ factors), the system performance could be improved further. Simulation results show that the proposed optimized adaptive WBAN system can achieve up to a 2- $\mathrm{dB}$ gain over the adaptive WBAN, thus providing
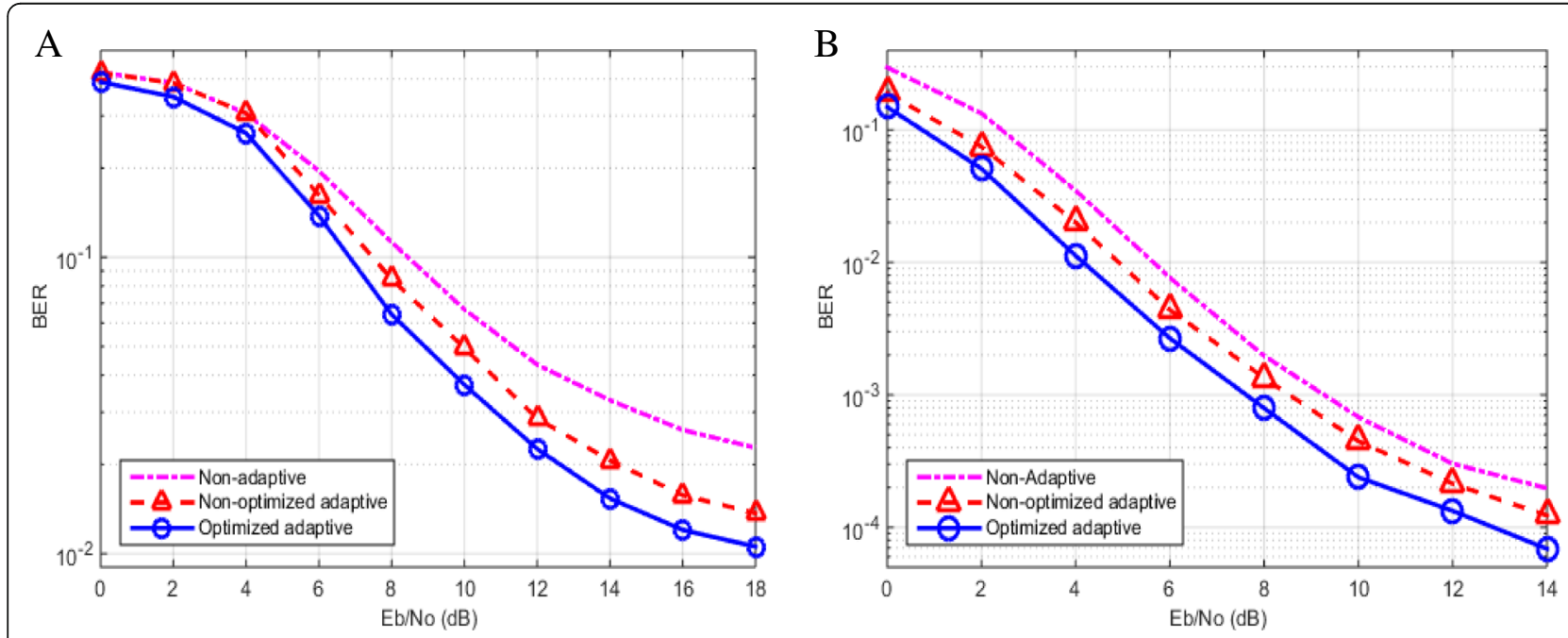

Fig. 11 Non-optimized adaptive and optimized adaptive performances a 2110 and b 2/2O 
up to a 6-dB gain over the non-adaptive one. This improvement is equivalent to a reduction of the transmission power by up to $37 \%$ compared to the nonoptimized adaptive WBAN, and up to $75 \%$ compared to the non-adaptive WBAN system. The improvements provide critical power reduction to WBAN devices, thus significantly extending the battery life and enhancing the reliability of WBAN services. Consequently, the proposed adaptive WBAN system could be one of feasible effective high-speed WBAN platform.

\section{Acknowledgements}

The first author acknowledges and is thankful to the financial support from AusAID through the Australian Development Scholarship (ADS) scheme. The authors are also grateful to the anonymous reviewers for the feedbacks.

\section{Competing interests}

The authors declare that they have no competing interests.

\section{Author details}

${ }^{1}$ School of Electrical, Computer, and Telecommunications Engineering (SECTE), University of Wollongong, Northfield Avenue, Wollongong New South Wales 2522, Australia. ${ }^{2}$ Department of Electrical and Computer Engineering, University of Nebraska, Lincoln, USA. ${ }^{3}$ University of Technology and Science, Bydgoszcz, Poland.

Received: 15 May 2016 Accepted: 10 November 2016 Published online: 25 November 2016

\section{References}

1. R Cavallari et al., A survey on wireless body area networks: technologies and design challenges. IEEE Communication Survey and Tutorial, vol. 99, 2014

2. M Chen et al., Body area networks: a survey. Springer J. Mobile Netw. Appl. 16(2), 171-193 (2010)

3. IEEE Computer Society, IEEE standard for local and metropolitan area networks-Part 15.6: Wireless Body Area Networks, 2012

4. A Batra et al., Multiband OFDM physical layer specification, WiMedia Alliance, Final Deliverable 1.5, 2009

5. Siriwongpairat, WP et al., Multiband-OFDM MIMO Coding Framework for UWB Communication Systems. IEEE Trans. Signal Process. 54, no. 1, 214-224 (2006)

6. TH Tan, KC Lin, Performance of space-time block coded MB-OFDM UWB systems, in Proc. IEEE CNSR-2006, 2006, pp. 323-327

7. Q Yang, KS Kwak, Outage performance of STBC MB-OFDM UWB. Int. J. Electron. Commun. 63(8), 685-688 (2008)

8. L.C. Tran, A. Mertins, Space-Time-Frequency Code implementation in MBOFDM UWB communications: design criteria and performance. IEEE Trans. Wireless Commun. 8, no. 2, Feb. (2009)

9. SM Alamouti, A simple transmit diversity technique for wireless communications. IEEE J. Sel. Areas Commun. 16(8), 1451-1458 (1998)

10. S Sezginer, H Sari, A high-rate full-diversity $2 \times 2$ space-time code with simple maximum likelihood decoding. Proc. ISSPIT-2007, 2007, pp. 1132-1136

11. LC Tran, A Mertins, TA Wysocki, Quasi-orthogonal space-time-frequency code in MB-OFDM UWB communications. J. Comput. Electrical Eng. 36(4), 766-774 (2010)

12. LC Tran et al., Novel constructions of improved square complex orthogonal designs for eight transmit antennas. IEEE Trans. Inf. Theory 55(10), 4439-4448 (2009)

13. H Sawada et al., Channel models between body surface and wireless access point for UWB band. IEEE P802.15-08-0576-00-0006, 2008

14. M Sudjai, LC Tran, F Safaei, Performance analysis of STFC MB-OFDM UWB in WBAN channels, in Proc. IEEE PIMRC-2012, 2012, pp. 1704-1709

15. KY Yazdandoost, K Sayrafian-Pour, Channel model for body area network (BAN). IEEE P802.15-08-0780-12-0006, 2010

16. SK Yoo et al., Shadowed fading in indoor off-body communications channels: a statistical characterization using the $\kappa-\mu / G a m m a$ composite fading model. IEEE Trans. Wireless Commun. 15(8), 5231-5244 (2016)

17. A Czylwik, Adaptive OFDM for wideband radio channel, in Proc. GLOBECOM2006, vol. 1, 1996, pp. 713-718
18. T Keller, L Hanzo, Adaptive modulation techniques for duplex OFDM transmission. IEEE Trans. Veh. Technol.49, no. 5, (2000)

19. T Keller, L Hanzo, Adaptive multicarrier modulation: a convenient framework for Time-Frequency processing in wireless communication. Proc. IEEE, 88, No.5, May (2000)

20. ST Chung, AJ Goldsmith, Degrees of freedom in adaptive modulation: a unified view. IEEE Trans. Commun. 49(9), 1561-1571 (2001)

21. S Fu et al., An enhanced link adaptation for the MB-OFDM UWB system, in Proc. IEEE WCNC-2012, 2012, pp. 2070-2075

22. J Garzas, CB Calzon, AG Armada, An energy-efficient adaptive modulation suitable for wireless sensor networks with SER and throughput constraints. EURASIP J. Wirel. Commun. Netw. 2007, 1-7 (2007)

23. WB Yang, K Sayrafian-Pour, Interference mitigation using adaptive scheme in body area networks. Int. J. Wireless Inf. Networks 19, 193-200 (2012)

24. JM Torrance, L Hanzo, Optimization of switching levels for adaptive modulation in slow Rayleigh fading. IEE Electron. Letters 32(13), 1167-1169 (1996)

25. A Argyriou, AC Breva, M Aoun, Optimizing data forwarding from body area networks in the presence of body shadowing with dual wireless technology nodes. IEEE Trans. Mob. Comput. 14(3), 632-645 (2015)

26. $\mathrm{N}$ Javaid et al., Adaptive medium access control protocol for wireless body area networks. Int. J. Distrib. Sens. Netw. 2014, 1-10 (2014)

27. AH Sodro, Y Lie, MA Shah, Energy-efficient adaptive transmission power control for wireless body area networks. IET Commun. 10(1), 81-90 (2016)

28. M Sudjai, LC Tran, F Safaei, A simple adaptive STFC MB-OFDM UWB system for WBAN applications, in Proc. IEEE ISCIT-2013, 2013, pp. 60-65

29. M Sudjai, LC Tran, A BER based adaptive STFC MB-OFDM UWB system for WBAN applications, in Proc. IEEE ICC-2014, 2014, pp. 5681-5686

30. M. Sudjai, et al., Optimal adaptive wireless body area networks for high speed mHealth services. Proc. $48^{\text {th }}$ DGBMT Annual Conference, Oct. 2014

31. A Milenkovic, C Otto, E Jovanov, Wireless sensor networks for personal health monitoring: Issues and an implementation. J. Comput. Commun. 2006(29), 2521-2533 (2006)

32. R Butt, Applied linear algebra and optimization using MATLAB (Mercury Learning and Information, Dulles Virginia, 2011)

33. D Bertsimas, R Weismantel, Optimization over integers (Dynamic Ideas, Belmont, MA, 2005)

34. M. Sandell, JP Coon, Per-Subcarrier antenna selection with power constraints in OFDM system. IEEE Trans. Wireless Commun. 8, no. 2, 673-677 (2009)

35. A Paulraj, R Nabar, D Gore, Introduction to space-time wireless communication, Cambridge University Press, Cambridge, 2008.

\section{Submit your manuscript to a SpringerOpen ${ }^{\circ}$ journal and benefit from:}

- Convenient online submission

- Rigorous peer review

- Immediate publication on acceptance

- Open access: articles freely available online

- High visibility within the field

- Retaining the copyright to your article

Submit your next manuscript at $>$ springeropen.com 